Anne van Aaken ${ }^{l}$

\title{
Massenklagen im öffentlichen Recht aus institutionenökonomischer Sicht: Eine Art der direkten Volksrechte
}

\author{
»Wer sein Recht behauptet, verteidigt innerhalb \\ des engen Raumes desselben das Recht ${ }^{2}$
}

\section{A. Einleitung}

Bedauerlicherweise leben wir nicht in der besten aller verwaltungsrechtlichen Welten und so ist denn auch die Kontrolle der Verwaltung ein seit langem diskutiertes und seit den Zweifeln an der Selbstlosigkeit der staatlichen Akteure ein besonders aktuelles Thema. ${ }^{3}$ Bei den Kontrollmechanismen der Verwaltung kann die selbstinitiierte von der fremdinitiierten Kontrolle unterschieden werden. Erstere ist die verwaltungsinterne Kontrolle, die aus Rechts- und Fachaufsicht durch übergeordnete Behörden, internen Personalkontrollen und neuerdings den Mechanismen des New Public Management besteht. Bei der fremdinitiierten externen Kontrolle finden sich die Rechnungshöfe, die parlamentarische Kontrolle, ${ }^{4}$ die Institution eines Ombudsmannes, formlose Rechtsbehelfe und vor allem auch die Kontrolle durch die (betroffenen)

1 Ich danke sehr herzlich Prof. Dr. Christoph Engel, Prof. Dr. Dieter Grimm, Thorsten Käseberg sowie Prof. Dr. Henner Kleinewefers für hilfreiche Anmerkungen und Hinweise.

2 So schrieb schon Jhering, Der Kampf um's Recht, 1880, 6. Aufl., S. 49 (Hervorhebung im Original).

3 Vgl. dazu Masing, Die Mobilisierung des Bürgers für die Durchsetzung des Rechts: europäische Impulse für eine Revision der Lehre vom subjektiv-öffentlichen Recht, 1997, der das Konzept des subjektiv-öffentlichen Rechts im deutschen, französischen und europäischen Recht behandelt und genau die Frage stellt, inwieweit dieses Konzept nur zur Durchsetzung privater Interessen des Bürgers in seiner Stellung als »bourgeois« oder auch zur Durchsetzung von öffentlichen Interessen gegenüber der Verwaltung genutzt werden kann und genutzt wird.

$4 \mathrm{Zu}$ der Kontrolle der Verwaltung durch das Parlament aus der Sicht der Neuen Institutionenökonomik, siehe Spiller, Politicians, Interest Groups, and Regulators: A Multiple-Principal Agency Theory of Regulation, or »let them be bribed«, Journal of Law and Economics 33, 1990, S. 65ff. sowie Weingast, The Congressional-Bureaucratic System: An Principal-Agent Perspective, Public Choice 44, 1984, S. 147ff., und im Zusammenspiel mit den Gerichten, siehe Spiller/Tiller, Decision Costs and the Strategic Design of Administrative Process and Judicial Review, Journal of Legal Studies 26, 1997, S. 347ff. sowie Smith/ Tiller, The Strategy of Judging: Evidence from Administrative Law, Journal of Legal Studies 31, 2002, S. 61ff., Tiller, Controlling Policy by Controlling Process: Judicial Influence on Regulatory Decision Making, Journal of Law, Economics and Organisation 14, 1998, S. 114ff. 
Bürger mittels Klagemöglichkeiten. ${ }^{5}$ Der Gedanke der Mobilisierung des Bürgers zur Durchsetzung des Rechts gewinnt dabei zunehmend an Bedeutung. ${ }^{6}$

Aus ökonomischer Sicht handelt es sich bei der Kontrolle der Verwaltung um ein Prinzipal-Agenten-Problem. ${ }^{7}$ In der Regel werden in der Neuen Politischen Ökonomie der Bürger als Prinzipal und die gewählten Politiker einer politischen Einheit als Agenten gesehen. Dies wird aber der Wirklichkeit nur begrenzt gerecht, da die Verwaltung als zweiter Agent sozusagen nachgeschaltet und in der Realität von großer Bedeutung ist. Um dieses delegierende Prinzipal-Agenten-Verhältnis zwischen Politik und Verwaltung nicht auszublenden, ist es sinnvoll, eine Agenten-Kette zu formulieren: Die Politiker werden von den Bürgern gewählt und kontrollieren ihrerseits die Verwaltung durch Gesetze (Kompetenzdelegation). Hinzu kommt bei einer solchen Betrachtung aber auch die Möglichkeit einer (zusätzlichen) direkten Kontrolle der Verwaltung durch die Bürger. Die Kontrolle der Verwaltung durch Massenklagen wird hier daher als alternative bzw. ergänzende Möglichkeit der Kontrolle der Politiker durch direkte Volksrechte auf legislativer Basis betrachtet. Die Bürger als Prinzipale kontrollieren dann nicht nur die Verwaltung als Agenten des Agenten, sondern mittelbar auch die gewählten Politiker, da inzident auch Legislativarbeit überprüft wird.

5 Diese Aussage gilt jedenfalls für das deutsche System, in dem der Rechtsschutz gegenüber der Verwaltung primär als verwaltungsgerichtliche (Ergebnis)Kontrolle ausgestaltet ist, während in USA das Schwergewicht der Kontrolle bei einem rechtsförmigen und fairen, waffengleichen Verfahren innerhalb der Verwaltung liegt. Vgl. dazu Scharpf, Die politischen Kosten des Rechtsstaats. Eine vergleichende Studie der deutschen und amerikanischen Verwaltungskontrollen, 1970, insbes. S. 14 sowie 38.

6 Vgl. ausführlich Masing, Die Mobilisierung des Bürgers, der einen »status procuratoris« des Bürgers (S. 225ff, 236), sowie Pernice, Gestaltung und Vollzug des Umweltrechts im Europäischen Binnenmarkt - Europäische Impulse und Zwänge für das deutsche Umweltrecht, NVwZ 1990, S. 414ff., 424, der einen »status activus processualis « vorschlägt.

7 Genauer gesagt handelt es sich bei diesem Prinzipal-Agenten-Problem um ein Problem des moralischen Risikos (»moral hazard«), also ein Problem nach Vertragsschluß. Dabei wird angenommen, dass die Information insoweit asymmetrisch ist, als 1) die Tätigkeit des Agenten vom Prinzipal nicht direkt beobachtet werden kann oder 2) der Agent Informationen hat, die der Prinzipal nicht besitzt. Außerdem ist es für den Prinzipal zu kostspielig, die Handlungen des Agenten direkt zu überwachen oder sich genaue Kenntnis der allein vom Agenten gewonnen Informationen zu verschaffen. Im ersten Fall spricht man von verstecktem Handeln, im zweiten von versteckter Information. Siehe dazu Richter/Furubotn, Neue Institutionenökonomik: eine Einführung und kritische Würdigung, 1996, S. 163. Im politischen Bereich kommt hinzu, dass der Prinzipal Entscheidungs- und Informationskompetenzen abgibt, also de iure die Möglichkeit der direkten Überwachung abgeschnitten wird. Der positive Prinzipal-Agenten-Ansatz beschäftigt sich primär mit der »Technologie « von Überwachung und Vertretungskosten des Agenten. Diesem Ansatz wird hier gefolgt, wenn von Prinzipal-Agenten-Problemen die Rede ist. Siehe dazu ausführlich Richter/ Furubotn, Neue Institutionenökonomik, S. 166ff. sowie Erlei/Leschke/Sauerland, Neue Institu tionenökonomik, 1999. 
In dem folgenden Schaubild soll verdeutlicht werden, wo dieser Artikel in der institutionenökonomischen Literatur seinen Platz hat:

\begin{tabular}{|c|c|c|}
\hline \multirow{4}{*}{$\begin{array}{l}\text { neu: Massenklagen } \\
\text { alt: Individualklagen }\end{array}$} & \multicolumn{2}{|l|}{ Bürger } \\
\hline & $\downarrow$ & $\begin{array}{l}\text { Prinzipal-Agent-Beziehung } \\
\text { (Wahlen/direkte Volksrechte) }\end{array}$ \\
\hline & Politiker & \\
\hline & $\downarrow$ & $\begin{array}{l}\text { Prinzipal-Agent-Beziehung } \\
\text { (Hierarchie) }\end{array}$ \\
\hline \multicolumn{3}{|c|}{ Prinzipal-Agenten-Beziehung im öffentlichen Recht (Abb. 1) } \\
\hline
\end{tabular}

Der Pfeil von den Bürgern zu den gewählten Politikern kennzeichnet Wahlen und eventuell direkte Volksrechte. Dies sind die gängigen Kontrollmöglichkeiten des Prinzipals gegenüber dem Agenten Legislative. Legislative bzw. Politiker überwachen ihrerseits die Verwaltung. Die Verwaltung handelt als »verlängerter Arm « der Legislative. ${ }^{8}$ Jedoch gibt es auch hier selbstverständlich Reibungsverluste, ${ }^{9}$ denn der immer größere Handlungsspielraum der Verwaltung ist kein bestrittenes Phänomen mehr. Der breite Pfeil bezeichnet die (direkten) Möglichkeiten der Bürger, die Verwaltung zu kontrollieren. Hierher gehören zunächst die bereits bestehenden individuellen Klagemöglichkeiten nach der VwGO. Zusätzlich kann Kontrolle mittelbar durch Einschaltung eines Ombudsmannes ausgeübt werden. ${ }^{10}$ Ebenso wird hier

8 Unterscheidet man mit Luhmann, Recht und Automation in der öffentlichen Verwaltung Eine verwaltungswissenschaftliche Untersuchung, 1966, S. 35-48, Konditional- und Zweckprogramme, so trifft die Aussage jedenfalls für die Konditionalprogramme zu, die nur reine Regelanwendung zulassen. Bei Zweckprogrammen wird der Verwaltung allerdings ein erheblich größerer Spielraum eingeräumt.

9 In der Begrifflichkeit der Neuen Institutionenökonomik: Residualverluste, die als Differenz zwischen dem hypothetischen Gewinn des Prinzipals bei einer neoklassischen »erstbesten Lösung « und dem tatsächlich anfallenden Gewinn bzw. Nutzen definiert ist. Der Wohlfahrtsverlust des Prinzipals entsteht durch Transaktionskosten und aufgrund von unzureichender Interessenvertretung durch den Agenten. Zu einer Übersicht über die PrinzipalAgenten-Thematik, siehe Breid, Aussagefähigkeit agencytheoretischer Ansätze im Hinblick auf die Verhaltenssteuerung von Entscheidungsträgern, Schmalenbachs Zeitschrift für betriebswirtschaftliche Forschung 9, Sonderdruck, 1995, S. 821ff.

10 Allerdings hat der Ombudsmann in der Regel keine rechtsprechende Funktion wie die Verwaltungsgerichte, vielmehr ist er eine Art informelle Beschwerdeinstanz für Schikanen und Verzögerungen im Verwaltungsverfahren. Zu einer rechtsvergleichenden kurzen Darstellung der Institution des Ombudsmannes, siehe Koch, Verbandsklage in Deutschland und Europa, ZZP 113, 2000, S. 413ff., 427f. und Koch, Prozeßführung im öffentlichen Interesse. Rechtsvergleichende Entwicklungsbedingungen und Alternativen objektiver Rechtsdurchsetzung, 1983, S. 80ff. Für die Schweiz siehe Knapp, Grundlagen des Verwaltungs- 
Verwaltungskontrolle durch Informationsrechte genannt. ${ }^{11}$ Massenklagemöglichkeiten wären eine weitere, effektivere Kontrollmöglichkeit, die aber in der ökonomischen Literatur in Deutschland nicht und in der juristischen Literatur primär im Zivilrecht thematisiert wird. ${ }^{12}$

Das deutsche Verwaltungsprozessrecht privilegiert bestehende subjektivrechtliche Positionen. Es diskriminiert hingegen solche Interessen, deren Subjekte die Allgemeinheit oder eine Vielheit von individuell nur geringfügig betroffenen Bürgern sind. Aber auch Massenschäden ${ }^{13}$ müssen rechtlich gelöst werden. Auf vielen Gebieten des täglichen Lebens lassen sich Normverletzungen beobachten, durch die gleichzeitig eine große Zahl von Bürgern beeinträchtigt wird, jeder einzelne für sich genommen aber nur einen so geringfügigen oder wenig greifbaren Nachteil erleidet, dass ihm die Rechtsordnung die Befugnis zur Erhebung einer Klage versagt oder dass eine Klage individuell nicht lohnend erscheint. Aber eine rein materiell-rechtliche Lösung im Sinne von Setzung objektiven Rechts ist notwendig geschwächt, wenn keine wirksamen Durchsetzungsmechanismen zur Verfügung stehen.

In der juristischen Literatur sind die prozeßrechtlichen Institute der Gruppenklage/ Verbandsklage/Popularklage ein seit langem diskutiertes Thema. Diese verschiedenen

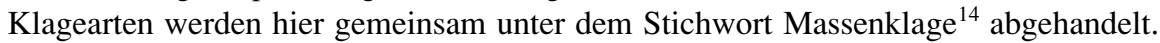
Massenklage wird als Gegenbegriff zur Individualklage verwendet. Logisch sind der

rechts, 1992, 4. Aufl., S. 456. Als Modell für Deutschland, siehe Franke, Ein Ombudsmann für Deutschland? Einführungsmöglichkeit einer Ombudsmann-Institution in das deutsche Verfassungsleben unter Berücksichtigung der dänischen und europäischen Einrichtungen, 1999.

11 Vgl. übersichtshalber Schoch, Informationsfreiheitsgesetz für die Bundesrepublik Deutschland, Die Verwaltung 35, 2002, S. 149ff. sowie ausführlich und unter Bezugnahme auf das UIG Schoch/Kloepfer (unter Mitwirkung von Garstka, Entwurf eines Informationsfreiheitsgesetzes für die Bundesrepublik Deutschland, 2002).

12 In der zivilrechtlichen Literatur sind hier Koch, Kollektiver Rechtsschutz im Zivilprozeß. Die Class Action des amerikanischen Rechts und deutsche Reformprobleme, 1976; Koch, Prozeßführung im öffentlichen Interesse; Koch, Alternativen zum Zweiparteiensystem im Zivilprozeß, KritV 1989, S. 232ff.; Koch, Die Funktionäre des Sozialschutzes im Prozeß. Ein rechtsvergleichender Überblick, KritV 1991, S. 386ff.; Koch, Verbandsklage in Deutschland und Europa, S. 413ff.; Becker, Verwaltungskontrolle durch Gesellschafterrechte: eine vergleichende Studie nach deutschem Verbandsrecht und dem amerikanischen Recht der corporation, 1997, sowie Hass, Die Gruppenklage: Wege zur prozessualen Bewältigung von Massenschäden, 1996, zu nennen.

13 Massenschäden sind solche, die bei einer Vielzahl von Personen aufgrund einer gleichartigen Ursache eintreten, siehe Hass, Die Gruppenklage: Wege zur prozessualen Bewältigung von Massenschäden, S. 20. Ob diese Schäden subjektive Rechte oder diffuse Interessen betreffen ist irrelevant. So liegt beispielsweise bei Waldschäden ein Massenschaden vor. $\mathrm{Ob}$ damit subjektive Rechte betroffen sind, wie bei den Eigentümern des Waldes (eine Klage gegen die Bundesregierung wegen unterlassener Umweltgesetzgebung wurde abgewiesen, BGHZ 102, 350), ist für die Definition von Massenschäden unbeachtlich.

14 Der Ausdruck Massenklage ist nicht mit dem Begriff des Massenverfahrens in der VwGO, etwa § 56a VwGO zu verwechseln und wird hier in Anlehnung an Röhl, Rechtssoziologie, 1987, S. 486 verwendet. Siehe zur sog. Treuhänderklage, die im Umweltschutz relevant ist, da »Eigenrechte « der Natur eingeklagt werden, siehe Koch, Die Funktionäre des Sozialschutzes im Prozeß, 386ff., S. 397f. 
materiell-rechtliche Anspruch und die prozeßrechtliche Klage unabhängig voneinander. ${ }^{15}$ So können etwa Individualschäden bei subjektiven Rechten mittels Individualklagen (Zwei-Parteien-Prozeß) oder aber auch durch Massenklagen (Gruppenklage) geltend gemacht werden. Ebenso ist es denkbar, dass Massenschäden in Individualklagen (Popularklage) geltend gemacht werden oder Massenschäden in Massenklagen behandelt werden (Verbandsklage).

Aus ökonomischer Sicht können Massenklagen als prozeßrechtliches Instrument der Internalisierung von massenhaft auftretenden (potentiellen) Externalitäten bezeichnet werden. Massenklagen ermöglichen die Zusammenfassung der atomisierten Einzelinteressen zu Gruppeninteressen und die Wahrnehmung öffentlicher Interessen seitens des Bürgers gegenüber der Verwaltung. Die Rechtmäßigkeit der Verwaltung ist ein öffentliches Gut. ${ }^{16}$ Es geht darum, den Bürger oder auch Verbände zu legitimieren und zu stimulieren, das Interesse der Allgemeinheit an der Rechtmäßigkeit der Verwaltung in bestimmten Bereichen und bei konkreten Sachproblemen vor einem neutralen Forum geltend zu machen. Dies ist besonders dringlich für das öffentliche Recht, da dort oftmals Sachverhalte bestehen, die eine Vielzahl von Bürgern betreffen und die nicht über den Markt (Privatrecht) gelöst werden können. Dieses öffentliche Gut »Verwaltungskontrolle« wird aber nicht bereitgestellt, weil es für den Einzelnen billiger ist, »Trittbrett zu fahren«, so dass das Gut nicht oder nicht ausreichend bereitgestellt wird.

Der vorliegende Aufsatz beschäftigt sich daher mit dem Ausbau und der Stimulation der privaten Kontrollmöglichkeiten der Verwaltung im öffentlichen Recht. Sind Gruppenklagen/Verbandsklagen/Popularklagen aus institutionenökonomischer Sicht sinnvoll? Welche Anreize für Individuen und Organisationen werden durch solche Klagearten vermittelt? Welche Kosten entstehen durch solche Klagearten und welchen Nutzen können solche Institute bringen? Tragen diese Klagearten zu einer wirksameren Kontrolle des Staates durch die Bürger bei? Welche funktionellen Ähnlichkeiten bestehen mit direkten Volksrechten und welche Vor- bzw. Nachteile werden von den Massenklagen geteilt?

15 Zwar ist es denkbar, dass das Problem der Massenschäden materiell-rechtlich gelöst wird, so wäre es jedenfalls bei manchen Sachverhalten möglich, Betroffenen subjektive Rechte zuzugestehen, jedoch sind andere Fälle denkbar, bei denen der Charakter des rein öffentlichen Gutes die Zuweisung von Eigentumsrechten verbietet. So könnte beispielsweise den Bewohnern in der Nachbarschaft eines Waldes ein subjektives Recht an der Erhaltung desselben eingeräumt werden (auf die dadurch entstehenden rechtlichen Probleme soll hier gar nicht weiter eingegangen werden), dasselbe ist aber nur schwerlich möglich bei der Erhaltung von sauberer Luft. Daher ist auch der von Ökonomen oft gemachte Vorschlag, Eigentumsrechte zu schaffen nur die Lösung für einen Teil der Probleme, vgl. auch Adler, Stand or Deliver: Citizen Suits, Standing, and Environmental Protection, Duke Environmental Law and Policy Forum 12, 2001, S. 39ff.

16 Öffentliche Güter sind durch nicht-rivalisierenden Konsum und durch Nichtausschliessbarkeit gekennzeichnet. Das Lehrbuchbeispiel ist saubere Luft. Zu derselben Thematik im Zivilrecht siehe grundlegend Jhering, Der Kampf um's Recht, sowie Kötz, Klagen Privater im öffentlichen Interesse, in: Arbeiten zur Rechtsvergleichung, Bd. 68, hg. von Homburger, 1975, S. 69-102, S. 72 mit rechtsvergleichenden Anmerkungen. 
Zur Beantwortung dieser Fragen ist es zunächst notwendig, die relevanten Begriffe aus der Jurisprudenz und der Ökonomik ${ }^{17}$ zu klären und die Anknüpfungspunkte zu finden, damit das methodische Instrumentarium der Ökonomik auf dieses juristische Problem angewendet werden kann. Dafür wird im ersten Teil ein Raster gebildet, welches es ermöglicht, die o.g. drei Klagearten sinnvoll gemeinsam zu analysieren. Im zweiten Teil wird dann das ökonomische Rationale für diese Klagearten dargelegt, indem die Anreizwirkungen solcher Institutionen untersucht werden. Hier wird auch die Parallele zu den direkten Volksrechten aufgezeigt.

\section{B. Rechte, Interessen, Klagerechte und Externalitäten: ein vergleichender juristisch- ökonomischer Ansatz}

Die Begrifflichkeiten von Juristen und Ökonomen sind unterschiedlich. Insbesondere wird der Begriff des öffentlichen Interesses oder Allgemeinwohls in der Regel nicht mit dem Begriff des Nutzens oder der Externalitäten zusammen gedacht. Zwischen diesen Begriffen besteht aber eine Verbindung, die im folgenden erklärt werden soll.

\section{Rechtlich geschützte Interessen: subjektive Rechte und diffuse Interessen}

In den kontinentaleuropäischen Rechtskreisen bestimmt die Unterscheidung zwischen subjektiven Rechten und diffusen Interessen gleichzeitig, welche Rechte bzw. Interessen durch begleitende prozessuale Möglichkeiten durchsetzbar sind und welche nicht.

Subjektiv-öffentliche Rechte umfassen zunächst die Grundrechte und grundrechtsgleichen Rechte. Eine Beeinträchtigung dieser Rechte eröffnet immer (und nur) dann eine Klagemöglichkeit, soweit der subjektiv-rechtliche Gehalt des Grundrechts betroffen ist. Es wird davon ausgegangen, dass der Einzelne sich bei Betroffenheit um einen (gerichtlichen) Schutz selber kümmert. Subjektiv-öffentliche Rechte ergeben sich gemäß der Schutznormlehre ${ }^{18}$ aber auch aus einfachen Rechtssätzen, wenn der in Frage stehende Rechtssatz als Normzweck nicht nur den Schutz des Allgemeininteres-

17 Ökonomie als Begriff wird in dieser Arbeit verwendet, um die Wissenschaft zu beschreiben, die sich mit wirtschaftlichen Zusammenhängen auseinandersetzt, also mit den traditionellen Gegenstandsbereichen der Wirtschaftswissenschaften, der Mikro- und Makroökonomie. Ökonomik wird als Begriff verwendet, wenn das Instrumentarium der Ökonomie auf nicht wirtschaftliche Zusammenhänge angewendet wird, also die allgemeine Theorie des menschlichen Verhaltens unter dem Aspekt der Knappheit betrachtet wird. Zu dieser Unterscheidung, siehe insbes. Kirchgässner, Homo Oeconomicus, 1991, S. 2f., der Ökonomik kurz als Methode und Ökonomie als Gegenstandsbereich der Wirtschaftswissenschaften bezeichnet.

18 So beispielsweise $\S 9$ I Nr. 1 und Nr. 24 BauGB und § 21 Abs. 1 BJagdG. Auf der Grundlage der herrschenden Schutznormtheorie (BVerfGE 27, 297 (307); BVerwG, Urteil vom 30. März 1995 - BVerwG 3 C 8/94; BVerwG Urteil vom 17. Juni 1993 - BVerwG 3 C 3.89 - in Buchholz 451.74 \& 10 Nr. 4 Seite 4; BVerwG, Urteil vom 16. März 1989 BVerwG 4 C 36.85 - in BVerwGE 81, 329 (334) sowie Urteil vom 16. Juni 1994 BVerwG 3 C 12.93) vermitteln Drittschutz nur solche Vorschriften, die nach dem in ihnen enthaltenen, durch Auslegung zu ermittelnden Entscheidungsprogramm auch der Rücksichtnahme auf die Interessen des betreffenden Dritten dienen. 
ses im Auge hat, sondern zumindest auch den Individualinteressen des potentiellen Klägers zu dienen bestimmt ist. Auf die Schutzwürdigkeit de facto kommt es dabei nicht an. Auch hier ist das rechtlich geschützte Interesse durch ein korrespondierendes individuelles Klagerecht geschützt. Der Einzelne ist berechtigt, bei Beeinträchtigung seiner Interessen den Rechtsweg zu beschreiten.

Diffuse Interessen ${ }^{19}$ sind rechtlich geschützte Interessen, die de lege lata nicht als subjektive Rechte qualifiziert werden und für die deswegen kein korrespondierendes prozessuales Recht existiert. Sie sind lediglich als objektives Recht staatlicherseits zu beachten. Unter einem dynamischen Aspekt betrachtet, sind es Interessen, über deren Subjektivierung und Klagbarkeit noch nicht entschieden ist. Auch die Berufung auf sogenannte Rechtsreflexe ist daher unzulässig. Das bonum commune dient dem Gesetzgeber und der Verwaltung als Argument für Eingriffe in bestehende Rechte zugunsten von an sich nicht individuell rechtlich geschützten Interessen. Es ist somit möglich, individuelle (potentielle oder diffuse) Interessen zum Allgemeinwohl zu aggregieren ${ }^{20}$ und so einen Eingriff zu rechtfertigen. Diese Möglichkeit hat allerdings nur der Staat. Individuen, auch wenn es viele sind, können sich bislang nicht auf ihre diffusen Interessen berufen und den Staat durch die Gerichte zum Handeln bewegen. ${ }^{21}$ Es gibt de lege lata kein prozeßrechtliches Äquivalent für diffuse Interessen, die als objektives Recht statuiert sind.

\section{Der ökonomische Begriff des Nutzens und der Externalität}

Für Ökonomen sind die Begriffe des Nutzens und der Externalitäten tägliches Brot. Welcher Zusammenhang besteht mit rechtlich geschützten Interessen? Der Nutzenbe-

19 Dieser Ausdruck stammt von Cappelletti, Access to Justice. A Comparative General Report, RabelsZ 40, 1976, S. 669ff., S. 680: »Diffuse interests are collective or fragmented interests such as that in clear air or the enforcement of consumers protection measures. The basic problem they present - the reason for their diffuseness - is that either no one has a right to remedy the infringement of a collective interest or the stake of any one individual in remedying the infringement is too small to induce him to seek enforcement action." Siehe zu dem Problem der diffusen Interessen und ihrer Organisationsmöglichkeit auch grundlegend Olson, Die Logik des kollektiven Handelns: Kollektivgüter und die Theorie der Gruppen, 1985, 2. durchges. Aufl. Siehe auch die Bezugnahme von Scharpf, Die politischen Kosten des Rechtsstaats, auf Olson, S. 68.

20 Seit dem Arrow'schen sog. (Un)möglichkeitstheorem wird von Ökonomen angenommen, dass es ein »Allgemeinwohl« nicht gibt, weil es bei Kollektiventscheidungen unter bestimmten Bedingungen unmöglich sein kann, konsistente Sozialwahlfunktionen zu finden, wenn diese u.a. auf ordinalen Präferenzen beruhen und nicht diktatorisch sein sollen. Siehe dazu Arrow, Social Choice and Individual Values, 1951, und Gäfgen, Theorie der wirtschaftlichen Entscheidung, 1974, 3. Aufl., S. 427ff. sowie zu sozialen Wohlfahrtsfunktionen Aaken, Rational-Choice in der Rechtswissenschaft. Zum Stellenwert der ökonomischen Theorie im Recht, 2003, Punkt 3.1.1.1. Dennoch wird das »Allgemeinwohl« oder das »öffentliche Interesse « weiterhin vom Gesetzgeber und der Verwaltung als unbestimmter Rechtsbegriff als Rechtfertigung für Eingriffe verwendet und muss dies auch.

21 In Ländern mit direkten Volksrechten ist es allerdings jederzeit möglich ein diffuses Interesse auf dem Wege der Gesetzes- oder Verfassungsinitiative in ein subjektives Recht zu überführen. 
griff der Ökonomen ist - in der Nachfolge des Utilitarismus - streng subjektiv definiert. Das ökonomische Konzept des Nutzens erlaubt es a priori, völlig unabhängig von der geltenden Rechtsordnung und den in ihr zugewiesenen Rechten, alle Interessen zu erfassen, also sowohl subjektive Rechte als auch diffuse Interessen und sogar rechtlich gar nicht geschützte Interessen. Analog erlaubt es der ökonomische Nutzenbegriff, alles, was individuell als Beeinträchtigung empfunden wird, als Nutzeneinbuße oder Kosten zu qualifizieren. Externalitäten sind solche Wirkungen von Entscheidungen oder Handlungen, bei denen die Kosten bzw. der Nutzen, die/der aufgrund der Handlung oder Entscheidung anfallen, nicht bei demjenigen anfallen, der die Entscheidung getroffen hat. Der Entscheidungsträger muß für die Konsequenzen seiner Handlung nicht aufkommen (negative Externalitäten bzw. externe Kosten) bzw. wird dafür nicht entschädigt (positive Externalitäten bzw. externer Nutzen). An der Entscheidung Beteiligte und von der Entscheidung Betroffene sind nicht identisch. Externe Effekte ${ }^{22}$ kommen zustande, weil Verfügungsrechte nur unzureichend spezifiziert oder die Transaktionskosten ${ }^{23}$ zu hoch sind, wodurch eine Handelbarkeit erschwert oder unmöglich ist. Sie stellen ein Marktversagen dar. Der individuelle Nutzen ist mit dem sozialen Nutzen nicht identisch, mit anderen Worten: Es gibt Individuen, deren Interessen nicht berücksichtigt werden.

Um externe Effekte über den Markt zu internalisieren, sind mehrere Bedingungen notwendig: zum einen müssen Verfügungsrechte ${ }^{24}$ genügend klar spezifiziert sein, zum zweiten muß es die (rechtliche) Freiheit geben, sie zu tauschen, ${ }^{25}$ zum dritten dürfen die Transaktionskosten den Transaktionsgewinn nicht übersteigen. Die Handelbarkeit von Verfügungsrechten sichert die Internalisierung der Effekte bei den beteiligten Vertragsparteien (nicht jedoch bei Dritten), da einer Nutzeneinbuße von einem rationalen Individuum freiwillig nicht zugestimmt würde (volenti-non-fit-iniuria-Prinzip), sondern ein Äquivalent dafür geboten werden muß, damit eine Zustimmung erfolgt. Weiterhin müssen diese Rechte durchsetzbar, also justiziabel sein.

22 Externe Effekte treten auch als sog. »pekuniäre« Externalitäten auf, nämlich solche, die durch das Preissystem vermittelt werden, also finanzielle Gewinne oder Verluste. Diese stellen regelmäßig kein Problem dar, weil sie auf Märkten entstehen und im üblichen Wege durch Marktentscheidungen verarbeitet werden können. Probleme entstehen nur bei den Externalitäten, die am Markt vorbei produziert werden. Allerdings gibt es natürlich auch hier implizit ein Werturteil, ob diese Externalitäten erwünscht sind, etwa im Wettbewerbsrecht.

23 Transaktionskosten i.w.S. sind Kosten der Koordination; auf den Markt bezogen also Kosten der Benutzung des Preismechanismus.

24 Unter Verfügungsrechten verstehen Ökonomen nicht nur Eigentumsrechte im Sinne von Art. 14 GG, sondern alle Handlungsrechte. Im weitesten Sinne umfassen sie die Zuordnung von Kompetenzen bei der Nutzung von Ressourcen. Alle denkbaren Arten von Rechten, auch Informations-, Entscheidungs-, Kontroll- und Klagerechte, aber auch diffuse Interessen werden damit umfaßt. Vgl. statt vieler zu einer extensiven Definition Schäfer/Ott, Lehrbuch der ökonomischen Analyse des Zivilrechts, 2000, 3. Aufl., S. 515.

$25 \mathrm{Zu}$ dem Problem der Handelbarkeit von bestimmten Verfügungsrechten, siehe grundlegend Calabresi/Melamed, Property Rules, Liability Rules, and Inalienability: One View of the Cathedral, Harvard Law Review 85, 1972, S. 1089ff. und Rose-Ackerman, Inalienability and the Theory of Property Rights, Columbia Law Review 85, 1985, S. $931 \mathrm{ff}$. 
Im Privatrecht können daher viele Arten von Externalitäten ${ }^{26}$ grundsätzlich über den Markt internalisiert werden. Zwar gibt es nicht für alle betroffenen Interessen ex ante spezifizierte subjektive Rechte, aber die Schaffung von subjektiven Rechten (property rights) ist durch privatautonomen Vertrag möglich. Dies wird als effiziente Lösung betrachtet, ${ }^{27}$ weil anzunehmen ist, dass der Kooperationsgewinn - zumindest zwischen den beteiligten Parteien - positiv ist.

Die Möglichkeiten der Internalisierung über den Markt im öffentlichen Recht sind begrenzt. Dies ist erstens darin begründet, dass im öffentlichen Recht die »Erhöhung« eines Interesses zu einem subjektiven Recht kraft Vertragsfreiheit nicht möglich ist. ${ }^{28}$ Zum zweiten liegt es daran, dass es zu viele Betroffene gibt, die nicht miteinander kontrahieren können, weil die Transaktionskosten zu hoch sind, weswegen der Staat als Agent für die vielen (bzw. zukünftig) Betroffenen handelt. Es handelt sich im öffentlichen Recht oftmals um Viel-Personen-Verhältnisse, bei denen die Internalisierung durch Zustimmung nicht gesichert ist. Drittens existieren Interessen, die den Charakter eines öffentlichen Gutes haben und daher nicht als subjektives Recht spezifizierbar sind. Es handelt sich zumeist um diffuse Interessen oder um Interessen, die erst mit einer gewissen Wahrscheinlichkeit in der Zukunft betroffen sind. Ein Beispiel sind Umweltschutzbelange, wobei allerdings auch hier versucht wird, handelbare Verfügungsrechte zu schaffen. ${ }^{29}$ In all diesen Fällen gibt es de lege lata nur objektives Recht, welches nicht durch den Einzelnen, sondern durch den Staat als Agent wahrgenommen wird.

26 Auch im Privatrecht gibt es allerdings Fälle von Externalitäten, die zwar subjektive Rechte betreffen, aber aufgrund zu hoher Transaktionskosten nicht durch Verträge internalisiert werden können. Hierzu zählen beispielsweise alle deliktsrechtlichen Fälle. In den Fällen, in denen die negative Externalität ein absolut geschütztes Rechtsgut betrifft, erhält das Individuum eine (je nach Schädiger) privat- oder öffentlich-rechtliche Klagemöglichkeit, durch welche bei Erfolg der Klage die geschaffene Externalität nachträglich durch Schadensersatzzahlungen oder Restitutionsansprüche internalisiert wird. Siehe dazu grundlegend Coase, The Problem of Social Cost, Journal of Law and Economics 3, 1960, S. 1ff.; Calabresi, The Costs of Accidents, A Legal and Economic Analysis, 1970; Coleman, Risks and Wrongs, 1992. Im Zivilprozeßrecht laufen daher die Probleme analog, siehe dazu Koch, Prozeßführung im öffentlichen Interesse; Koch, Alternativen zum Zweiparteiensystem im Zivilprozeß, S. 232ff; Koch, Verbandsklage in Deutschland und Europa, S. 413ff.

27 Effizienz gemäß dem Pareto-Kriterium besagt, dass eine Situation A besser ist als eine Situation B, wenn es in A mindestens einem Individuum besser und keinem Individuum schlechter geht als in B. Das Pareto-Kriterium betrachtet den Nutzen der beteiligten Individuen in der Situation A im Vergleich mit der Situation B. Es sichert bei allen Beteiligten die Internalisierung von externen Effekten. Externalitäten, die nicht von den Beteiligten erwünscht sind, und Situationen, denen sie deswegen nicht zustimmen, genügen dem Pareto-Kriterium daher nicht. Allerdings gibt es häufig Situationen, in denen es Betroffene gibt, die nicht beteiligt werden und somit aus der Betrachtung herausfallen. Das ParetoKriterium wird in der Ökonomik und Ökonomie als relativ wenig kontroverses Kriterium verwendet und reicht für die hiesige Analyse aus.

28 Eine begrenzte Ausnahme bildet der öffentlich-rechtliche Vertrag gemäß $\S 54 f f$. BVwVfG.

29 So etwa die Verschmutzungszertifikate in Art. 3 Abs. 12 des Kyoto-Protokolls (http:// www.bmu.de/download/dateien/protodt.pdf), siehe dazu auch »The Economist« v. 22.01.2000, S. 68f. 
Wie kann mit solchen Externalitäten umgegangen werden, die entweder nicht über den Markt internalisiert werden können, also Externalitäten, bei denen aus technischen Gründen keine handelbaren Eigentumsrechte geschaffen werden können, oder die nicht über den Markt internalisiert werden sollen? Wenn eine Beteiligung aller Betroffenen durch ihre Zustimmung nicht möglich ist, kann es statt dessen entweder eine Internalisierung qua Gesetz, qua behördliche Entscheidung und/oder eine Internalisierung mittels gerichtlicher Entscheidung geben. Fraglich ist dann aber, welche Externalitäten vom Staat beachtet werden. De lege lata ist klar: auch diffuse Interessen werden berücksichtigt, aber nur im objektiven Recht. Dieses ist aber grundsätzlich für Bürger nicht einklagbar. Damit schließt sich dann die Frage an, ob eine Beteiligung von Betroffenen (Prinzipal) durch Klagemöglichkeiten gegeben sein sollte. ${ }^{30}$ Denn die Klagemöglichkeit ist ebenfalls eine denkbare Internalisierungstrategie, wenn die Verwaltung nicht oder nur unzureichend tätig wird und die Internalisierung (bei erfolgreicher Klage) stattdessen gerichtlich angeordnet wird.

\section{Eine ökonomische Begründung für Massenklagen im öffentlichen Recht}

Wie gesehen, korrespondiert mit den subjektiven Rechten das prozeßrechtliche Äquivalent des Zwei-Parteien-Prozesses. Bei der Betroffenheit von diffusen Interessen oder objektivem Recht handeln dagegen nur die staatlichen Stellen als Agenten »im Namen« der betroffenen Individuen. Denkbar wäre aber natürlich auch, Klagerechte und damit Überwachungsrechte bei den Bürgern als dem Prinzipal zu belassen und so die externe Kontrolle der Verwaltung und auch der Legislative zu verbessern. Massenklagen haben insofern funktionelle Ähnlichkeit mit direkten Volksrechten, insbesondere der Initiative. Ökonomisch kann begründet werden, warum es sinnvoll ist, bei der Verwaltungskontrolle »direkte Volksrechte« in der Form der Massenklagen zuzulassen.

Zunächst wird das ökonomische Rationale, das für Massenklagen im Allgemeinen auf individueller und gesellschaftlicher Ebene gilt, dargestellt. Die funktionellen Parallelen zwischen Massenklagen und direkten Volksrechten unter Einbeziehung der diskutierten Vor- und Nachteile werden im Allgemeinen aufgezeigt. Sodann werden die Argumente gesondert und detailliert für Gruppenklagen, Verbandsklagen und Popularklagen im Besonderen betrachtet.

\section{I. Ökonomischer Sinn von Massenklagen im Allgemeinen}

Die Besonderheit des Agenten Staat liegt darin, dass er ein (Gebiets-)Monopol innehat, woraus folgt, dass eine Qualitätskontrolle durch den Markt bzw. durch Konkurrenz nicht stattfindet. Abwanderung oder Widerspruch (nicht im verwaltungsrechtlichen Sinne) sind die Reaktionsmöglichkeiten des Bürgers bei schlechter

30 Auch hier kann unterschieden werden, ob eine Klagemöglichkeit für die Begünstigten im Wege der Schutznormtheorie geschaffen wird, oder aber, ob alle (potentiell) Betroffenen eine Klagemöglichkeit durch Änderung des Prozessrechts eingeräumt bekommen. Hier kann auch wieder zwischen den verschiedenen Arten der Massenklage unterschieden werden, die jeweils andere Klagebefugte zulassen. 
Qualität. ${ }^{31}$ Abwanderung ist in der Regel kostspielig, weshalb Widerspruch und die Schaffung der rechtlichen Möglichkeit dafür, d.h. entsprechende Klagemöglichkeiten, sinnvoll erscheinen. Wie in der ökonomischen Literatur dargelegt, kann die Kontrolle des Agenten durch den Prinzipal Wohlfahrtsverluste des Prinzipals, die durch das Vertretungsverhältnis entstehen, senken. Wenn eine Aufgabe der Verwaltung Internalisierung von Externalitäten ist, so ist die Annahme sinnvoll, dass Externalitäten solange $\mathrm{zu}$ beseitigen sind, wie die Grenzkosten der Internalisierung die Grenzkosten der Externalität nicht übersteigen. Wenn zweifelhaft ist, ob dieses Optimum erreicht ist, muß gefragt werden, welche rechtlichen Wege dafür zur Verfügung stehen. ${ }^{32}$ Zur Beurteilung muss nach den realen Auswirkungen des geltenden Prozeßrechts gefragt werden, d.h. sowohl nach den Anreizwirkungen für das Individuum, als auch nach den gesellschaftlichen Auswirkungen. ${ }^{33}$

\section{Anreizstrukturen für das Individuum für die Erhebung von Massenklagen}

Die Anreize eines Individuums die Verwaltung durch Klagen zu kontrollieren, können bei rein rational-theoretischer ${ }^{34}$ Betrachtungsweise identifiziert werden. Der Nutzen des Individuums zu klagen wird eng definiert, nämlich als individueller Internalisierungsnutzen. Betrachten wir zunächst die Anreizstrukturen des Prozeßrechts für ein von einer Externalität betroffenes Individuum in der folgenden Graphik:

$31 \mathrm{Zu}$ den Mechanismen Abwanderung und Widerspruch (»voice«) siehe grundlegend A.O.Hirschman, Abwanderung und Widerspruch, 1974. Der »Voice-Mechanismus" kommt bei der Verwaltung nur begrenzt zum Tragen. Anders ist es bei der Abwanderung, die, wenn sie rechtlich und faktisch möglich ist, einen Wettbewerb zwischen den Gebietskörperschaften herbeiführt, siehe grundlegend dazu Tiebout, A Pure Theory of Local Expenditures, Journal of Political Economy 64, 1956, S. 416ff.

32 Ein Optimum definiert immer einen Punkt, in dem sich zwei Prinzipien die Waage halten, also nicht ein Prinzip maximiert wird. Bei der Verwaltungskontrolle bedeutet dies, dass immer auch dem Nutzen einer solchen die Kosten derselben gegenüberstehen. Scharpf, Die politischen Kosten des Rechtsstaats, spricht denn auch in seiner rechtsvergleichenden Studie (deutsches und US-amerikanisches Verwaltungsrecht) von den politischen Kosten des Rechtsstaates bzw. von den Spannungen zwischen Rechtsstaat und demokratischer Politik. Scharpf, S. 70ff., diagnostiziert in einer vorweggenommenen präzisen Normierung des Verwaltungsprogramms eine Verminderung der Qualität der verfügbaren Informationen. Folgt man dieser These, so müsste aber folgerichtig die ex ante Kontrolle durch die Legislative durch eine ex post Kontrolle durch die Gerichte (durch Massenklagen) kompensiert werden.

$33 \mathrm{Zu}$ dem Verhältnis des Sozialschutzes, der Legitimation und der Bevormundung bei staatlicher Repräsentation, Gruppenklagen, Verbandsklagen und Popularklagen, siehe Koch, Die Funktionäre des Sozialschutzes im Prozeß, S. 386ff., 398ff.

34 Es sei unbestritten, dass andere als rationale Erwägungen eine Rolle spielen können, aber dies gilt nicht im Durchschnitt - und nur dieser ist für die Gesetzgebung primär relevant. Zudem sei beachtet, dass die $45^{\circ}$-Linie der Einfachheit halber Risikoneutralität annimmt bei Risikoaversion bzw. Risikofreudigkeit wäre die Linie entsprechend konvex bzw. konkav. 


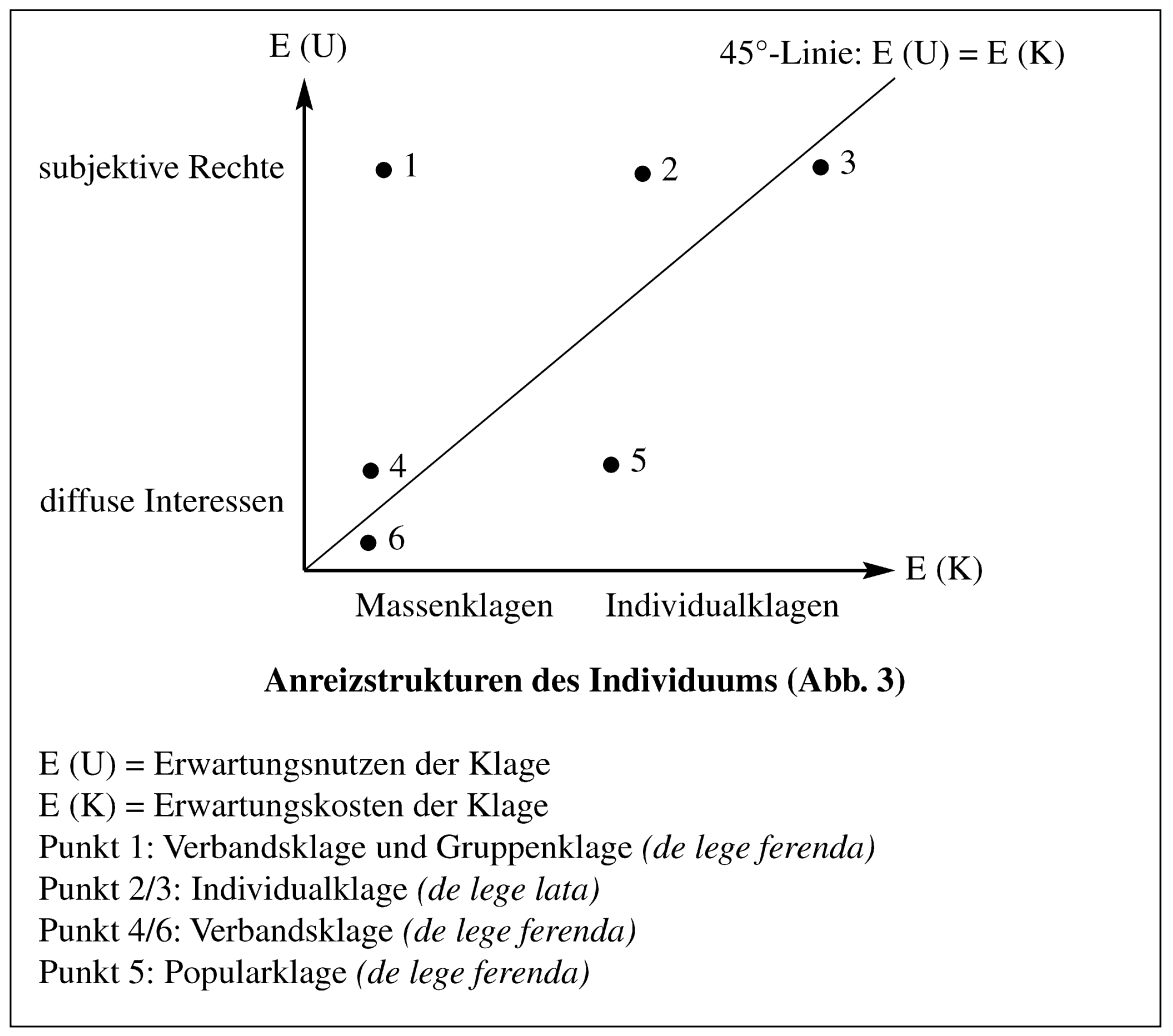

Auf der Abszisse finden sich die erwarteten Kosten der Klage. Auf der Ordinate findet sich der erwartete Nutzen der Klage. Ein Individuum wird, so die Ökonomik, dann und nur dann klagen, wenn der erwartete Nettonutzen der Klage positiv ist, also bei allen Punkten oberhalb der $45^{\circ}$ - Linie. Bei allen Punkten unterhalb der Linie ist der erwartete Nettonutzen negativ, weswegen eine Klage unterbleiben wird.

Der erwartete Nutzen der Klage ergibt sich aus den Erfolgschancen der Klage und dem Nutzen aus dem nunmehr zu beachtenden Interesse. Letzteres ist die Internalisierung der beklagten Externalität durch gerichtliche (stattgebende) Entscheidung. ${ }^{35} \mathrm{Je}$ größer das beeinträchtigte Interesse bzw., ökonomisch gesprochen, die (negative) Externalität ist, desto größer wird der Nutzen aus der Internalisierung sein. Die Externalität kann sowohl in der Beeinträchtigung eines subjektiven Rechts bestehen als auch in der Beeinträchtigung eines diffusen Interesses, wenn dieses als ausgedünntes Verfügungsrecht betrachtet wird, d.h. der Nutzen entsteht entweder durch die Wiederherstellung des subjektiven Rechts oder die Beachtung des diffusen Interesses. Hier

35 Die Internalisierung durch die angeordnete Rechtsfolge kann verschieden aussehen, je nachdem, ob es sich um Schadensersatzforderungen, Unterlassungsansprüche oder Leistungsansprüche handelt. 
wird die Annahme getroffen, dass die Beeinträchtigung eines subjektiven Rechts eine stärkere Beeinträchtigung des Individuums darstellt als die Beeinträchtigung eines diffusen Interesses. Dies ist verständlich, weil es sich in der Regel um absolut geschützte Rechtsgüter wie beispielsweise Leben, Handlungsfreiheit, Eigentum (Grundrechte) handelt. Auch die durch Schutznormen geschützten subjektiven Rechte fallen darunter. Annahmegemäß ist der Nutzen bei ihnen nicht so hoch wie bei den Grundrechten. Allerdings kann auch die Beeinträchtigung von subjektiven Rechten so geringfügig sein, dass eine Klage unterbleibt. ${ }^{36}$ Das Rationale für die diffusen Interessen gilt daher analog für die geringfügigen Einzelschäden. Damit wird auch deutlich, dass es sich um ein Kontinuum des individuellen Nutzens handelt.

Die erwarteten Kosten des Individuums sind bei Individualklagen typischerweise größer als bei Massenklagen, da das Individuum bei ersteren alle Kosten, also sowohl erwartete Anwalts- und Gerichtskosten ${ }^{37}$ als auch alle anderen zu erwartenden materiellen und immateriellen Kosten, alleine tragen muss. Die immateriellen Kosten fangen an mit der Suche nach einer anwaltlichen Vertretung, Verständigungsschwierigkeiten und hören nicht auf mit dem Zeitverlust, der durch einen solchen Prozess entsteht. ${ }^{38}$ Die direkten materiellen Kosten des Verfahrens können unterschieden werden in Kosten des eigenen Rechtsbeistandes und von Gutachtern sowie das Risiko der Verurteilung zur Zahlung der Gerichtskosten. Der Erwartungswert der Prozesskosten richtet sich (bei dem geltenden Alles-oder-Nichts-Prinzip der Kostentragung ${ }^{39}$ ) nach den Erfolgschan-

36 Darunter fallen die Fälle, die in USA durch die »class action« gelöst werden, vgl. dazu ausführlich Koch, Kollektiver Rechtsschutz im Zivilprozeß. Die Class Action des amerikanischen Rechts und deutsche Reformprobleme.

37 Die Gerichtskosten richten sich nach dem Streitwert, welcher sich gem. § 13 Abs. 1 S. 2 GKG grundsätzlich nach der sich aus dem Antrag des Klägers für ihn ergebenden Bedeutung der Sache nach Ermessen des Gerichts richtet. Zur Streitwertbemessung, siehe Streitwertkatalog für die Verwaltungsgerichtsbarkeit: Fassung 1996, NVwZ 1996, S. 563ff. sowie Zimmer, Entwicklung des Streitwertes in der Verwaltungsgerichtsbarkeit seit 1988, NVwZ 1991, S. 547ff.

38 Siehe dazu Raiser, Rechtssoziologie, 1987.

39 In den USA gilt demgegenüber grundsätzlich das Alles-Oder-Nichts-Prinzip oder auch »Verlierer zahlt alles«-Prinzip nicht; dort bleiben die Anwaltskosten grundsätzlich bei den Prozessbeteiligten - ungeachtet der gerichtlichen Entscheidung - liegen, womit dieser Einflussfaktor in den USA wegfällt. Die unterliegende Prozesspartei zahlt in den USA grundsätzlich nur die Gerichtskosten. Siehe Kötz, Klagen Privater im öffentlichen Interesse, S. 73. Die Begründung dafür ist, dass das Recht des Bürgers auf gerichtlichen Rechtsschutz beeinträchtigt würde, müsste er das Risiko der Tragung der gegnerischen Anwaltskosten beachten. Bekannte Ausnahmen bestehen im Kartellrecht, S. 4 des Clayton Act (Gedanke des privaten Klägers im öffentlichen Interesse), im Umweltrecht und im Civil Rights Act. Alle diese Regelungen greifen nur zugunsten des erfolgreichen Klägers ein, nicht zugunsten des erfolgreichen Beklagten. Siehe zu weiteren Hinweisen sowie Nachweisen zur Kritik an der US-amerikanischen Kostentragungsregel, siehe Kötz, Klagen Privater im öffentlichen Interesse, S. 75 und Krent, The Fee Shifting Remedy: Panacea or Placebo?, Chicago-Kent Law Review 71, 1995, S. 415ff. Zu den Fragen, ob die US-amerikanische Gebührenregelung eine conditio-sine-qua-non für Verbands- und Gruppenklagen sind, siehe Koch, Prozeßführung im öffentlichen Interesse; Koch, Verbandsklage in Deutschland und Europa, S. 413ff. 
cen der Klage. ${ }^{40}$ Bei Individualklagen sind alle diese Kosten höher, erstens weil bei der Massenklage die Anwaltskosten sowie alle übrigen Kosten geteilt werden und zweitens weil davon auszugehen ist, dass bei vereinter und gebündelter Finanzkraft und Kompetenz der Anwaltskanzlei ${ }^{41}$ oder der Prozessbeteiligten (zum Beispiel der Verbände) auch die Erfolgschancen der Klage erhöht werden, ${ }^{42}$ was aber wiederum bei dem (deutschen) Alles-oder-Nichts-Prinzip die erwarteten Anwalts- und Gerichtskosten vermindert. Die erwarteten Kosten der Klage variieren mit den verschiedenen Klagearten und den Kostentragungsregeln. Die Kosten sind daher ein Kontinuum.

Betrachten wir das Anreizsystem des Individuums anhand der Punkte in Abb. 3 im Detail. Bei der Beeinträchtigung von subjektiven Rechten wird das Individuum ceteris paribus eher als bei der Beeinträchtigung von diffusen Interessen bereit sein zu klagen, da die Nutzeneinbuße durch die Beeinträchtigung des Rechts größer, mithin also auch der Nutzen aus einer gewonnenen Klage höher ist (Punkte 1, 2 und 3). Zudem kann davon ausgegangen werden, dass durch die Entscheidung in dem Prozess nur ein privates Gut bereitgestellt wird, da die Klage nur inter partes wirkt und daher alle Kosten internalisiert werden. Erst wenn die erwarteten Kosten der individuellen Klage sehr hoch werden, wird eine Klage unterbleiben (Punkt 3). Bei diffusen Interessen sind die Entscheidungen des Gerichts ein öffentliches Gut, da eine Vielzahl von Betroffenen diese diffusen Interessen teilen. Bei öffentlichen Gütern gibt es erhebliche Anreizprobleme, diese bereitzustellen, da das Individuum, wenn es nicht eine sehr hohe Präferenzintensität für das öffentliche Gut hat, aus rationaler Erwägung Trittbrett fahren wird; es wird mithin darauf warten, dass andere das Gut bereitstellen (und dafür zahlen), (vgl. Punkt 2 und Punkt 5). ${ }^{43}$ Wenn die Kosten der Klage hoch sind, dann ist zu erwarten, dass eine individuelle Klage unterbleibt (Punkt 5). Werden allerdings die erwarteten Kosten gesenkt durch die Möglichkeit einer Massenklage, so ist die Wahrscheinlichkeit der Klage höher (Punkt 4). Nur diffuse Interessen, deren Beeinträchti-

40 Es existiert allerdings die Möglichkeit der Streitwertherabsetzung. Im Zivilrecht findet sich diese Möglichkeit interessanterweise bei den Verfahren, bei denen in der Regel eine Vielzahl von Interessen betroffen ist, so im Patentgesetz (PatG), im Aktiengesetz (AktG) und im Gesetz gegen unlauteren Wettbewerb (UWG).

$41 \mathrm{Zu}$ dem Prinzipal-Agenten-Problem zwischen Klient und Anwalt und den Anreizstrukturen von Anwälten siehe Dewatripont/Tirole, Advocates, Journal of Political Economy 107, 1999, S. 1ff. Zu dem Verhältnis von Klient und Anwalt aus kombinierter spieltheoretischer Sicht und Prinzipal-Agenten-Betrachtungsweise, siehe auch Gilson/Mnookin, Cooperation and Competition in Litigation: Can Laywers Dampen Conflict?, in: Barriers to Conflict Resolution, hg. von Arrow, 1995, S. 184-211 m.w.N. Hier soll nur darauf hingewiesen werden, dass in USA die Anreizstrukturen von Anwälten wegen der Zulässigkeit von Erfolgshonoraren anders sind und auch mehr direkten Wettbewerb bringen. Siehe zu der Kritik insbesondere im Zusammenhang mit »class action « Mykkeltvedt, Common Benefits and Class Actions: Eliminating Artificial Barriers to Attorney Fee Awards, Georgia Law Review 36, 2002, S. $1149 \mathrm{ff}$.

$42 \mathrm{Zu}$ einer umfassenden Übersicht über die Einflussfaktoren auf die Erfolgsbarrieren in der Justiz, siehe Bender, Einige Aspekte zu den Erfolgsbarrieren in der Justiz, RabelsZ Bd. 40, 1976, S. 718ff., 726. Er nennt denn auch die Gleichheit aller Verfahren für verschiedenste Konflikte »ideologischen Popanz«, S. 718.

43 Siehe dazu Kirsch, Neue Politische Ökonomie, 1997, 4. überarb. u. erw. Aufl., S. 148ff. 
gung als gering empfunden wird, werden auch durch Massenklagen nicht gerichtlich überprüft (Punkt 6).

Bei gleichbleibender Beeinträchtigung kann daher davon ausgegangen werden, dass es eher bei der Möglichkeit der Massenklage als bei einer Individualklage zu einer Klage kommen wird, da der erwartete Nettonutzen der Klage bei der Massenklage wegen der geringeren erwarteten Kosten größer ist (vgl. Punkt 1 und 2 mit Punkt 3 für subjektive Rechte und Punkt 4 und 5 für diffuse Interessen). Daraus folgt auch, dass, bei gleichen erwarteten Kosten der Klage E (K), das Individuum eher bereit ist, bei der Beeinträchtigung eines subjektiven Rechts zu klagen als bei der Beeinträchtigung eines diffusen Interesses, weil der Nutzengewinn aus einer erfolgreichen Klage gegenüber dem status quo ante höher ist. Dies wird deutlich in dem Unterschied zwischen Punkt 1 und Punkt 6 einerseits und Punkt 2 und Punkt 5 andererseits. Bei den jeweils letzteren wird eine Klage unterbleiben, weil es sich um diffuse Interessen handelt, welche bei der alleinigen Möglichkeit der Individualklage nicht eingeklagt werden. Anders ist es aber, wenn Massenklagen zulässig wären; dann wird die Wahrscheinlichkeit, dass auch bei der Beeinträchtigung von diffusen Interessen geklagt wird, erhöht, wie man an Punkt 4 erkennen kann. Dies gilt im Durchschnitt, denn da der Nutzen subjektiv definiert ist, hängt es von der subjektiven Nutzeneinbuße des Individuums ab, ob es klagen wird oder nicht. Die Präferenzintensität des Individuums für das beeinträchtigte Interesse kann also durchaus einen Unterschied machen, wie bei der Popularklage (Punkt 5) noch zu zeigen sein wird. ${ }^{44}$

Fassen wir zusammen: Massenklagen verringern die Hürden, die sowohl rechtlich als auch tatsächlich aufgrund der bestehenden Anreizstrukturen eine wirksame Kontrolle der Verwaltung durch den Bürger behindern. Das rationale Desinteresse des Bürgers an der Internalisierung und dadurch mittelbar der Kontrolle der Verwaltung wird durch die Möglichkeit von Massenklagen, insbesondere Gruppen- und Verbandsklagen, gemildert. ${ }^{45}$

\section{Gesamtgesellschaftliche Begründung von Massenklagen}

Die Argumente auf gesamtgesellschaftlicher Ebene für die Einführung von Massenklagen werden im folgenden aufgeführt: ${ }^{46}$

1) Bei Massenschäden handelt es sich nicht um sinnvoll abgrenzbare Individualbelastungen, sondern um einen diffusen individuellen Anteil an einem Schaden, der eine

44 Die Inanspruchnahme der actio popularis ist geringer, als bei der Einführung dieser Klageart in beispielsweise USA erwartet und befürchtet wurde. Durch das obige Schema kann der Grund jedoch gut erklärt werden. Zudem ist die Verwendung der Popularklage geringer als die Verwendung der Verbandsklage oder der Gruppenklage, vgl. Groenendijk, Die Wahrnehmung gebündelter Interessen im Zivilprozeß, ZfRSoz Vol.2, 1982, S. $240 f f ., 265$.

$45 \mathrm{Zu}$ der rationalen Apathie, Schäden einzufordern, wenn der Schaden gering ist, siehe Schäfer/Ott, Lehrbuch der ökonomischen Analyse des Zivilrechts, S. 337f.

46 Die Begründung von Massenklagen im öffentlichen Recht bewegt sich auf der Ebene der sozialen Gegebenheiten, die Argumente sind also hier ökonomische und rechtstatsächliche. Die juristischen Argumente widersprechen den Realitäten der faktischen Betroffenheit der Bürger. 
ganze Gruppe von Menschen trifft. Die Art des Interesses, nämlich die Geringfügigkeit des betroffenen Rechts, verhindert individuelle Klagen.

2) Das bei vielen Gesetzen beklagte Implementations- und Vollzugsdefizit könnte verringert werden. Die Einführung von Massenklagen würde schon wegen ihres »Drohpotentials« die Einhaltung der Gesetze durch die Verwaltung fördern. Durch die wirksamere Kontrolle ist eine bessere Umsetzung des Rechts zu erwarten: einerseits besteht ein Anreiz für die Verwaltung, die Betroffenen frühzeitig in den Entscheidungsprozess mit einzubeziehen, um potentielle Prozesse zu vermeiden, und andererseits werden volkswirtschaftliche Kosten vermieden, indem weniger Schäden nachträglich behoben bzw. ausgeglichen werden müssen. ${ }^{47}$ Das US-amerikanische Beispiel im Umweltschutz zeigt, dass allein die potentielle Klagemöglichkeit die Behörden dazu anhält, von vornherein die betroffenen Belange genauer zu prüfen, Konflikte zu minimieren und außerhalb förmlicher Verfahren zu lösen, mit anderen Worten die Betroffenen werden frühzeitig in den Entscheidungsprozess einbezogen. Der Bedarf an administrativen Festlegungen und Kontrollen wird verringert, weil Widersprüche und Klagen eher unterbleiben.

3) Die Transparenz der Verwaltung würde durch den Offenlegungszwang vor Gericht erhöht. Dies vermindert das »moral-hazard «-Problem zwischen Bürger als Prinzipal und Verwaltung als Agent durch Milderung der Informationsasymmetrie und erhöht zudem die Akzeptanz der Verwaltungsentscheidungen. ${ }^{48}$ Dieser Vorteil kann allerdings auch bereits durch ein Informationsfreiheitsgesetz erreicht werden. ${ }^{49}$

4) Die Klagen der Bürger sind aber nicht nur als gegen die Verwaltung gerichtet zu betrachten, vielmehr sind sie ebenso als Informationsmittel für die Verwaltung dienlich, da durch solche Klagen (oder schon im Vorfeld) auf übersehene Probleme aufmerksam gemacht werden kann. ${ }^{50}$ Wegen der direkten Betroffenheit der Bürger, also einem »Näher-Dran-Prinzip«, bietet sich der Ausbau der verwaltungsrechtlichen Klagemöglichkeiten an. Im Privatrecht und insbesondere im Deliktsrecht handelt der Bürger als »privater Staatsanwalt «, indem er selbst die Schäden, die entstehen, prozessual geltend macht. ${ }^{51}$ Der Bürger handelt dort als »Agent « für den Staat, indem er für die

47 Oder wie Rehbinder, Argumente für die Verbandsklage im Umweltrecht, ZRP Heft 7, 1976, S. 157ff., 160, es bezüglich von Verbandsklagen ausdrückt: »Sie verursacht soziale Kosten - um größere soziale Kosten abzuwenden.«

48 So heißt es denn auch schon bei Kant: »Alle auf das Recht anderer Menschen bezogenen Handlungen, deren Maxime sich nicht mit der Publizität verträgt, sind ungerecht.« Kant, Zum ewigen Frieden, 1965, S. 145.

49 Vgl. dazu ausführlich Schoch/Kloepfer (unter Mitwirkung von Garstka), Entwurf eines Informationsfreiheitsgesetzes für die Bundesrepublik Deutschland.

50 Dieses Ziel kann allerdings grundsätzlich auch durch Partizipationsrechte (etwa von Umweltschutzverbänden) erreicht werden, wenn die Nichteinhaltung wie etwa in § 29 Abs. 1 i.V.m. § 4 Abs. 3 BNatSChG sanktioniert ist. Siehe zu den bereits existierenden Beteiligungsmöglichkeiten, die das Konzept der rein privatnützigen Wahrnehmung von Rechten relativieren, Masing, Die Mobilisierung des Bürgers, S. 128-174.

$51 »(\mathrm{~T})$ he victim acts as a private prosecuter bringing an action not only on his own behalf but also as an «agent « of the state. The state has an interest in discouraging economically inefficient behavior, but has only limited resources for doing so. By providing an avenue through which victims can secure resources for harm done to them, the state creates an 
Beachtung der Rechtsordnung sorgt. Im öffentlichen Recht fehlt eine solche Kontrolle für alle nicht als subjektives Recht ausgestalteten Interessen.

5) Oftmals handelt es sich bei den Massenschäden um Folgen technischer Großprojekte, bei denen den Individuen der technische Sachverstand fehlt, also eine eindeutige Informationsasymmetrie herrscht. Für die Einleitung eines Verfahrens ist nicht nur die Verfügbarkeit der erforderlichen technischen Information ein entscheidender Faktor. Auch der Mangel an Information über ein Rechtsverfahren an sich und über die einschlägigen materiell-rechtlichen Rechtsnormen bildet ein Prozeßhindernis. Dieses kann durch die Umlegung der Informationskosten auf die Gesamtheit der Betroffenen gemildert werden.

6) De facto stehen dem Individualkläger oft genug Wirtschaft und Verwaltung als finanzkräftige Gegner gegenüber. Die für eine Klage aufzuwendenden finanziellen Ressourcen übersteigen das Vermögen eines Einzelnen oftmals, wodurch eine Ungleichheit in den erwarteten Kosten des Verfahrens geschaffen wird. ${ }^{52}$

7) Bereits aus prozeßökonomischen Gründen wäre es sinnvoll, die Klagen in einem Verfahren zusammenzufassen. Warum eine Streitgenossenschaft wegen der individuellen Anreizwirkungen Defizite aufweist, wird bei den Gruppenklagen besprochen (Punkt II).

Welche Einwände werde typischerweise gegen Massenklagen eingebracht?

1) Der »korrekte« Weg der Kontrolle der Verwaltung gehe vom Wähler über das Parlament. Massenklagen als Weg über die Gerichte seien ein Verstoß gegen die Gewaltenteilung und daher nicht hinnehmbar. Die damit verbundene Politisierung der Gerichte sei unerwünscht und mit ihrer Funktion nicht vereinbar. Zudem seien sie häufig auch nicht dafür kompetent. Dem sollen zwei Argumente entgegengestellt werden: Erstens: die Verwaltung wird auch jetzt bereits durch die Gerichte kontrolliert, wenn auch direkt nur bei Verletzung subjektiven Rechts, welches eine »gate-keeping « Funktion einnimmt. Dieses ist somit zwar Einstieg in die Prüfung, aber nicht deren Maßstab, denn durch die Inzidenzkontrolle werden die Rechtsgrundlagen immer mitgeprüft. Die Gerichte kontrollieren also weiterhin nur das, was sie auch kontrollie-

institution of »private enforcement «. The expectation (or hope) of compensation induces private prosecutions, necessary to secure the optimal mix of private and public enforcement. « Coleman, Bilateralism, in: The Practice of Principle. In Defense of a Pragmatist Approach to Legal Theory, hg. von Coleman, 2001, S. 13-24., S. 19. Auch Koch, Die Funktionäre des Sozialschutzes im Prozeß, S. 395 redet bei den Popularklagen von einem »privaten Staatsanwalt « bzw. »private attorney general «.

52 Bei empirischen Untersuchungen im Zivilrecht wird deutlich, dass erstens Organisationen gegenüber Individuen strategische Vorteile bei der Prozeßführung haben und dass zweitens häufige Prozessierer eine vorteilhaftere Position im Prozeß haben als einmalige »Spieler«. Siehe dazu Groenendijk, Die Wahrnehmung gebündelter Interessen im Zivilprozeß, S. 240ff., S. 241 m.w.N. Im öffentlichen Recht kann angenommen werden, dass dieses Problem nochmals verstärkt wird, da die Verwaltung erstens eine stabile Organisation ist, zweitens in der Regel mehr Prozeßerfahrung hat als der Prozeßgegner und drittens in der Regel Informationsasymmetrie herrscht. 
ren sollen, nämlich die Einhaltung von Recht und Gesetz. ${ }^{53}$ Es ergibt sich keine qualitative Änderung in der Gewaltenteilung. Zweitens: die Gerichte, insbesondere die Obergerichte, sprechen auch jetzt Recht mit politischen Implikationen, die Gewaltenteilung ist de facto lange nicht so streng, wie oftmals vorgebracht wird.

2) Der freiheitliche und autonome Bürger sei selbst in der Lage, seine Rechte wahrzunehmen. Der klassische Liberalismus vertraut darauf, dass sich jedes verletzte Interesse vor Gericht artikulieren kann und daher automatisch ein optimaler Interessenausgleich herbeigeführt wird. Die Einräumung von Befugnissen nicht notwendigerweise betroffener Individuen (Popularklage) und Organisationen (Verbandsklage) führe zu einem Eingriff in diese Freiheit und zur Bevormundung. Dabei wird stillschweigend angenommen, dass es überall eine genügende Anzahl von klagebereiten Individuen gibt. Dieser Harmonieoptimismus darf als übertrieben bezeichnet werden, denn dies ist - aus individuell rationalen Gründen - gerade nicht der Fall, wie oben gezeigt werden konnte. Es gibt schwer zu organisierende allgemeine Interessen, deren rechtswidrige Verletzung keine Sanktion auslöst, weil sie unübersehbar Viele, diese Vielen aber jeweils nur geringfügig, bloß mittelbar oder in einer aus anderen Gründen nur schwer faßbaren Form betrifft. Man mag einwenden, dass es sich bei den typischen Fällen von Massenschäden um Gemeinwohlinteressen allgemeinsten Zuschnitts handele, zu deren Schutz der Staat und nicht der einzelne Bürger berufen sei. Und in der Tat wäre die Sorge um diese Interessen und damit die Zulässigkeit privater Klagen zur (objektiven) Rechtskontrolle unbegründet, wenn man darauf vertrauen dürfte, dass die Verwaltungsbehörden diese Interessen in der erforderlichen Weise wahrnehmen würden. Jedoch besteht die Gefahr, dass die Verwaltungsbehörden diese Allgemeininteressen aus politischen und wirtschaftlichen Rücksichten nicht ausreichend berücksichtigen, trotz der rechtlich bestehenden Eingriffsmöglichkeiten. ${ }^{54}$ 3) Die Zulassung von Massenklagen ermögliche eine unzulässige Einmischung in die Rechte und Lebensverhältnisse Dritter. Bedeutung erlangt dieser Einwand insbesondere bei (durch Massenklagen erleichterten) Drittwiderspruchsklagen. Doch auch hier wird seitens des Gerichts nur das geprüft, was die Verwaltung in ihr Ermessen hätte einstellen müssen. Das Problem der Verlängerung von Genehmigungsverfahren (und folgender Investitionshemmnisse) beispielsweise soll hier nicht verschwiegen werden. Dennoch erscheint die rechtliche oder faktische Behinderung der Durchsetzung eines von der Rechtsordnung anerkannten Rechtes oder Interesses nicht der richtige Weg, vielmehr müssen die Verwaltungsentscheide einer Überprüfung zugänglich sein. ${ }^{55}$

53 Siehe Masing, Die Mobilisierung des Bürgers, S. 93ff. zu der mittelbaren Geltendmachung objektiv-rechtlicher Normen, da der Bürger innerhalb der gezielten Eingriffsverwaltung die Verletzung jeglicher Vorschriften rügen kann.

54 Siehe Kötz, Klagen Privater im öffentlichen Interesse, S. 96, mit weiteren, auch empirischen Nachweisen.

$55 \mathrm{Zu}$ weiteren Argumenten, siehe Rehbinder, Argumente für die Verbandsklage im Umweltrecht, S. 162. 
4) Es sei eine Überforderung der Verwaltung und der Gerichte durch Prozeßüberflutung zu befürchten. ${ }^{56}$ Dies wird aber, aufgrund der oben aufgezeigten Anreizstrukturen, höchstens auf mutwillige Kläger und Querulanten zutreffen, vielmehr ist nur möglicherweise von einer quantitativen Zunahme der Klagen auszugehen. Ansonsten geht es nur um die Überprüfung der Rechtmäßigkeit von Verwaltungshandeln, welche jedenfalls zu fördern ist. Demgegenüber steht aber die Prävention von noch größeren Schäden, entweder durch »Generalprävention« bei der Verwaltung oder durch Gerichtsentscheid. Ökonomisch gesprochen sind Massenklagen solange zu fördern, wie die (sozialen) Grenzkosten der Rechtsdurchsetzung (etwa Kosten der Gerichte) kleiner sind als der Grenznutzen der Rechtsdurchsetzung. Eine Pauschalablehnung von Massenklagen ist somit nicht aufrechtzuerhalten.

3. Massenklagen als eine Form der direkten Volksrechte

Direkte Volksrechte werden in der ökonomischen Literatur als mögliche Lösung des Prinzipal-Agenten-Problems dargestellt. ${ }^{57}$ Direkte Volksrechte, also Volksinitiativen und Referenden, sind aus ökonomischer Sicht eine Art der externen Kontrolle des Agenten Staat im Allgemeinen und der Politiker im Besonderen. Sie umfassen die Kontrolle der Legislative, nicht aber die legislative Tätigkeit der Exekutive, ${ }^{58}$ also keine Rechtsverordnungen und Allgemeinverfügungen. ${ }^{59}$ Auch Einzelfallentscheidungen der Exekutive können durch direkte Volksrechte nicht beeinflußt werden. Die Kontrolle der Verwaltung durch Massenklagen wird daher hier alternativ und ergänzend zu der Kontrolle der Politiker durch direkte Volksrechte betrachtet. Massenklagen bewirken nur eine gerichtliche Kontrolle, während Volksrechte direkt legislative Änderungen herbeiführen.

56 Dies ist empirisch nicht dargelegt, im Gegenteil zeigen die Erfahrungen mit der Popularklage in USA eher das Gegenteil, siehe Kötz, Klagen Privater im öffentlichen Interesse, S. 101 m.w.N. Siehe auch Schoch, Informationsfreiheitsgesetz, S. 162 zu der entgegen den Prognosen nicht eingetretenen Flut von Anfragen bei dem UIG und den entsprechenden Landesgesetzen.

57 Siehe dazu mit der Darstellung der Argumente der Befürworter und der Gegner von direkten Volksrechten auf Bundesebene in der Schweiz: Kleinewefers, Die direkten Volksrechte in der Schweiz aus ökonomischer Sicht. Ein Überblick, in: Wieviel direkte Demokratie verträgt die Schweiz?, hrg. von Borner/Rentsch, 1997, S. 61-92, insbes. S. 62ff.

58 Diese Beeinflussung der legislativen Tätigkeit der Exekutive durch direkte Volksrechte spielt nur indirekt, wenn nämlich die entsprechende Delegationsnorm, die referendumsfähig sein muß, angegriffen wird, eine Rolle. Eine Ausnahme bildet das Finanzreferendum in der Schweiz.

59 Auch wenn die Allgemeinverfügung einen Verwaltungsakt gemäß § 35 S.2 BVwVfG darstellt, so ist sie doch funktional aufgrund ihres generell-konkreten Charakters als legislative Tätigkeit einzuordnen. 
Überblickshalber wird die Überprüfung von Rechtsgrundlagen (Gesetzen) und Verwaltungshandeln durch direkte Volksrechte einerseits und Massenklagen andererseits in folgender Graphik dargestellt:

\begin{tabular}{|l|l|l|}
\hline Überprüfung durch/von & $\begin{array}{l}\text { Rechtsgrundlage } \\
\text { (Legislative) }\end{array}$ & $\begin{array}{l}\text { Verwaltungshandeln } \\
\text { (Verwaltung) }\end{array}$ \\
\hline $\begin{array}{l}\text { direkte Volksrechte } \\
\text { (Bürger) }\end{array}$ & $\begin{array}{c}\text { I }+++ \\
\text { Referendum }\end{array}$ & II --- \\
\hline $\begin{array}{l}\text { Massenklagen } \\
\text { (Bürger und Gerichte) }\end{array}$ & $\begin{array}{c}\text { III +++ } \\
\text { inzident: Gruppen-/ } \\
\text { Verbandsklagen } \\
\text { direkt: Popularklagen }\end{array}$ & IV +++ \\
\hline
\end{tabular}

Mittel der Überprüfung des Staatshandelns (Abb. 4)

Einerseits bewirken Entscheidungen in Massenklagefällen Veränderungen nur innerhalb des rechtlichen Systems. Daher unterliegt ihr Veränderungspotential engeren Restriktionen als dasjenige von direkten Volksrechten. Sie geben nur geringeren Veränderungsspielraum, weil sich die Gerichte an das geltende Recht halten müssen. Bei Popularklagen, die sich gegen materielle Gesetze richten, aber auch bei Verbandsund Gruppenklagen gegen Verwaltungsakte und Allgemeinverfügungen werden die gesetzlichen Rechtsgrundlagen des Verwaltungshandelns inzident auf ihre Verfassungsmäßigkeit geprüft. Wenn die Rechtsgrundlage, wie im Regelfall, rechtmäßig ist, so beschränkt sich die Wirkung von Massenklagen auf die Überprüfung und gegebenenfalls die Korrektur des Verwaltungshandelns (Quadrant IV). Bei Zweifeln an der Verfassungsmäßigkeit der gesetzlichen Grundlage kann das Gericht eine konkrete Normenkontrolle beim Verfassungsgericht anstrengen. Bei der Feststellung der Verfassungswidrigkeit des Gesetzes hätte die Klage dann gesetzesaufhebende Wirkung (Quadrant III). In diesem Fall wirken Massenklagen funktional wie Ablehnungsinitiativen, weil nur eine gesetzesaufhebende, aber keine gesetzesschaffende Wirkung hervorgebracht wird.

Andererseits sind Massenklagen bezüglich des Prüfungsumfanges des Agenten Staat umfassender als die direkten Volksrechte. Letzere bewirken nur eine Kontrolle der Gesetze über den Stimmbürger (nicht durch die Gerichte). Erstere dagegen bewirken nicht nur die Kontrolle der Gesetze durch die Gerichte, sondern auch der Ausführung der Gesetze durch Verwaltungshandeln, jedenfalls soweit die behördlichen Entscheidungen gerichtlich überprüfbar sind. Um mit Scharpf zu sprechen: »das verwaltungsgerichtliche Urteil interpretiert die diffuse Gesetzesnorm mit Präjudizwirkung, es konkretisiert das Verwaltungsprogramm und bindet die ausführenden Behörden in allen künftigen Anwendungsfällen. ${ }^{60}$ Solche Einzelfallentscheidungen von Behörden können aber in Sachfragen große Wirkungen haben. Die Kontrolldichte

60 Scharpf, Die politischen Kosten des Rechtsstaats, S. 55. 
der Verwaltung ist also bei Massenklagen grundsätzlich höher als bei direkten Volksrechten. Wo liegen die Parallelen und die Unterschiede von Massenklagen und direkten Volksrechten aus ökonomischer Sicht und welche Argumente gegen direkte Volksrechte würden die Massenklagen nicht oder nur vermindert treffen? Zur Beantwortung dieser Fragen werden die Massenklagen auf der Basis des Evaluationsschemas von Kleinewefers ${ }^{61}$ zu direkten Volksrechten untersucht.

a) Internalisierung in der Politik: Prinzipal-Agenten-Probleme und Minderheiten Ein wichtiger Aspekt innerhalb der Prinzipal-Agenten-Beziehung ist die Bestimmung der politischen Agenda: Welche Probleme werden überhaupt im politischen Prozeß thematisiert? Die mangelnde oder gar fehlende Artikulation der Probleme von schwer organisierbaren Interessen und Minderheiten spiegelt sich in der Vernachlässigung auf der Agenda wider. Andere Probleme dagegen sind auf der politischen Agenda prominent, weil sie etwa durch mächtige Interessengruppen auf die politische Tagesordnung gesetzt werden. Zudem bleiben Politik und Verwaltung oftmals untätig oder übersehen bestimmte Interessen, insbesondere wenn diese sowieso im Hinblick auf die politischen Verhältnisse wenig Durchsetzungschancen oder Stimmpotential zu haben scheinen. ${ }^{62}$ Befürworter der direkten Volksrechte machen daher geltend, dass das PrinzipalAgenten-Problem zwischen Bürger und Staat reduziert würde, wenn und weil die Bürger das »agenda-setting« mitbestimmen könnten. Dem wird u.a. entgegengehalten, dass die Interessengruppen, und zwar die Kartelle der dominierenden Gruppen, gestärkt würden. Minderheiten würden noch weniger gehört.

Ein weiterer Aspekt des Prinzipal-Agenten-Problems ist das Ausmaß der Problemorientierung der Entscheidungen. Denn nicht nur die politische Agenda, sondern auch die politischen Entscheidungen werden im Hinblick auf auftauchende Probleme sachlich und zeitlich spezifiziert. Da über konkrete Fragen, nicht mehr nur über Paketlösungen abgestimmt wird, machen die Befürworter der direkten Volksrechte geltend, dass damit auch eine erhöhte Legitimation und Akzeptanz der Bürger zu erwarten sei. Dies sei darauf zurückzuführen, dass die Spezifikation eine Anpassung der politischen Entscheidungen an die Präferenzen der Bürger zur Folge habe.

Diese Argumente wollen wir im folgenden im Hinblick auf ihre Validität bezüglich der Massenklagen untersuchen. Massenklagen lassen »agenda-setting« von Bürgern auch in kleinerem Rahmen und problemspezifisch zu, allerdings nur in dem Rahmen, der von der Legislative bereits gesetzt ist. ${ }^{63}$ Der Kritik an dem übermäßigen Einfluß

61 Kleinewefers, Die direkten Volksrechte in der Schweiz aus ökonomischer Sicht. Dieses Schema enthält die Beurteilungskriterien, die hier in den folgenden Unterpunkten besprochen werden.

62 Dies ist insbesondere dann der Fall, wenn ein bestimmtes Thema nur mit Mühe auf die »political agenda« gesetzt werden kann, weil es als Detailfrage, eine komplizierte Frage oder eine typische Rechtsfrage abgestempelt wird.

63 Das »agenda-setting « wird natürlich mitbestimmt von den Interessengruppen und bei der Verbandsklage insbesondere von den Verbänden. Es ist richtig, dass die großen Verbände insofern ein Machtübergewicht haben. Jedoch ist gerade die Verbandsklage auch für kleine Verbände eine Chance, zu Wort zu kommen, da Klagen billiger sind als Referenden oder Initiativen. Dennoch kommt es entscheidend auf die Ausgestaltung der Zulassung und Anerkennung von Verbänden an. Bei der Gruppenklage ist das US-amerikanische Beispiel 
der großen Interessenverbände würde insofern Rechnung getragen, weil nun auch Minderheiten Artikulationsmöglichkeiten haben. Mehr noch als die direkten Volksrechte verstärken Massenklagen eine Spezifizierung bzw. Punktualisierung von Entscheidungen. Die Spezifikation im Hinblick auf bestimmte Sachprobleme nimmt zu, weil problembezogen geklagt wird. Auch hier ist daher eine Annäherung der Verwaltungsentscheide an die Präferenzen der Betroffenen zu erwarten. Zwar geht es zumeist eher um Sachfragen als um rechtspolitische Entscheidungen, weil die Klagen in der Regel das geltende Recht nicht verändern. Dennoch erlauben Massenklagen, Sachfragen und Rechtsfragen zu behandeln, da beide von den Gerichten überprüft werden. Diese Überprüfung hat den weiteren Vorteil, dass sie alle Betroffenen umfaßt, unabhängig von der politischen bzw. föderalistischen Organisation des Staates. Es sind Klagen denkbar, die beispielsweise Betroffene aus zwei oder mehreren Kommunen betreffen, bzw. spill-over-Effekte thematisieren. Eine Klage könnte diese Probleme zusammenfassen, ohne dass in der jeweiligen einzelnen Körperschaft Gesetze geändert werden müßten. Massenklagen sind bezüglich der Betroffenheit präziser als die legislative Problembehandlung in den jeweiligen staatlichen Körperschaften ${ }^{64}$ und erhöhen so die Möglichkeit der Internalisierung. ${ }^{65}$

b) Stärkung der öffentlichen Debatte

Ein weiteres Beurteilungskriterium ist die durch Volksrechte induzierte Veränderung der politischen Diskussion. ${ }^{66}$ Die Befürworter der direkten Volksrechte nehmen eine Stärkung der politischen Debatte durch vermehrte Konkurrenz bei dem Angebot der »politischen Ware« an. Politische Informationen würden erstens billiger (von den Interessengruppen) und zweitens öfters (nicht nur zu Wahlen) zur Verfügung gestellt. Dem wird entgegengehalten, dass erstens die Information nicht immer ausgewogen und von Qualität sei und dass es zweitens zu einem »information-overflow « käme, der nicht mehr verarbeitet werden könne.

aufschlußreich. Die Gruppenklagefälle in den USA werden oftmals zu einer Frage der Politik, wie sich beispielhaft an den Bluterfällen und der Gruppenklage der amerikanischen Juden gegen die Schweiz belegen läßt.

64 Siehe dazu Eichenberger, Der Zentralisierung Zähmung. Die Föderalismusdiskussion aus politisch-ökonomischer Perspektive, in: Öffentliches Recht als Gegenstand ökonomischer Forschung, hg. von Engel/Morlok, 1998, S. 157-173, S. 157ff.

65 In den Fällen der Verletzung subjektiven Rechts, in denen Massenklagen die Bündelung der atomisierten Externalitäten erlauben, wie bei Gruppenklagen, kommt es de facto eher zu einer Internalisierung, da die Klagehürden in der Form von Kosten und Informationsdefiziten gesenkt werden. Hier wird also eine de iure bestehende, aber de facto nicht genutzte Internalisierungsmöglichkeit durch Gerichte nur effektiver genutzt werden. Vermehrt gilt dies für Popularklagen und Verbandsklagen: Die Thematisierung der Sachprobleme werden durch »billigere« Klagemöglichkeiten erleichtert und die Internalisierung durch Beteiligung der Betroffenen wird wahrscheinlicher.

66 Dies ist in der Demokratietheorie und der »social-choice-theory « ein prominentes Thema. Siehe dazu Aaken, Deliberative Institutionenökonomik oder: Argumentiert der homo oeconomicus? Entwurf zu einer Kombination von Neuer Institutionenökonomik und Diskurstheorie, in: Ökonomie als Sozialwissenschaft, hrg. von Ötsch/Panther, 2002, S. 211-251 sowie Elster (Hg.), Deliberative Democracy, 1998. 
Massenklagen verstärken die politische Debatte punktuell und sachbezogen, nämlich dort, wo eine Klage anhängig ist oder angestrengt werden soll. Die Klagemöglichkeit erhöht den Anreiz, auf konkrete Probleme aufmerksam zu machen, die bislang nicht bewußt waren oder nicht beachtet wurden. ${ }^{67}$ Ein Informationsüberfluß ist dagegen bei der Einführung von Massenklagen nicht zu erwarten, weil eben die Informationen nur dann an die Öffentlichkeit gelangen und für diese relevant sind, wenn ein Bedürfnis aufgrund von Betroffenheit dafür besteht.

c) Systemkompatibilität und Innovationsfähigkeit

Eine weiterer Diskussionspunkt bezüglich direkter Volksrechte ist die Systemkompatibilität. Zwei Systemeigenschaften werden aus den Wirkungen der direkten Volksrechte abgeleitet und diskutiert: Innovationsfähigkeit und dynamische Stabilität des Systems.

Die Befürworter der direkten Volksrechte diagnostizieren eine vermehrte Innovationsfähigkeit des Systems, insbesondere durch die (Gestaltungs-)Initiative, da die Bürger änderungsfreudiger seien als die Politiker und zudem die Probleme schneller aufgegriffen und in die politische Diskussion getragen würden. Das System sei stabiler, weil laufend inkrementelle Verbesserungen vorgenommen würden, was Entwicklungsbrüche vermeide. Referenden dagegen wirkten eher bremsend, da die Legislative nicht mehr so schnell handeln könne. Nun ist aber zweifelhaft, ob erstens diese Innovationsfähigkeit insgesamt tatsächlich vorliegt und zweitens ob sie überhaupt erwünscht ist. Die Gegner diagnostizieren eher eine besondere Trägheit des Systems aufgrund des Konservativismus des Volkes, wodurch dann wiederum Entwicklungsbrüche provoziert würden. Zudem bestünde die Gefahr der punktuellen Interventionen, deren Systemwirkungen nicht bedacht würden.

Die verfassungsrechtliche Systemkompatibilität der Massenklagen ist wegen der notwendigen Verfassungskonformität der gerichtlichen Entscheidungen zu bejahen. Auch die einfachgesetzliche Kompatibilität ist grundsätzlich zu bejahen, da die Einführung von Massenklagen in aller Regel (anders nur die Popularklage) keine Änderungen im materiellen Recht herbeiführen, sondern sich vielmehr auf die Umsetzung des Rechts durch die Verwaltung beziehen. Die Systemveränderung ist langsamer und weniger grundlegend als bei direkten Volksrechten. Die Innovationsfähigkeit von Massenklagen ist im Vergleich mit direkten Volksrechten insoweit begrenzter. Dies ist wiederum auf ihr beschränktes Veränderungspotential zurückzuführen. Jedoch können im Einzelfall zumindest auf Mikro- und Mesoebene Verbesserungen zustande kommen. Anders ist die Innovationsfähigkeit nur dann zu beurteilen, wenn durch eine solche Massenklage das Verfahren vor das BVerfG gelangt, da dann, bei Feststellung der Verfassungswidrigkeit des Gesetzes, gesetzesändernde Wirkung auftritt. Der Einwand des unberechenbaren und punktuellen Interventionismus wird bei Massenklagen insofern entkräftet, als die Entscheidungen sich innerhalb des rechtlichen Systems halten müssen. Punktuell sind die Entscheidungen allerdings, aber das liegt in der inter par-

67 Dies gilt insbesondere bei der Verbandsklage für Verbände oder bei der Popularklage für politische Unternehmer, die ein Interesse daran haben, die Klage und die zugrundeliegenden Probleme in die Öffentlichkeit zu tragen. 
tes-Natur der Verwaltungsverfahren. Inwieweit Fern- und Nebenwirkungen bei einem solchen Verwaltungsverfahren beachtet werden, kann hier nicht im Einzelnen diskutiert werden. Sicher ist aber, dass durch Massenklagen mehr Informationen in die Verwaltungsverfahren getragen werden, so dass zumindest von einer Tendenz zu einer vermehrten Berücksichtigung dieser Wirkungen ausgegangen werden kann.

Zusammenfassend läßt sich feststellen, dass Massenklagen das Prinzipal-AgentenProblem zwischen Staat und Bürger bezüglich der Verwaltung entschärfen können. Ebenso kann die öffentliche Debatte sachproblembezogen gestärkt werden. Das Reformpotential ist im Vergleich mit direkten Volksrechten geringer. Sie teilen aber viele Vorzüge der direkten Volksrechte und schneiden in punkto Systemkompatibilität und Berechenbarkeit besser ab. ${ }^{68}$

\section{II. Ökonomischer Sinn von Gruppenklagen}

Die Gruppenklage (auch Sammelklage genannt) ist eine Art des Prozesses, bei der eine Vielzahl von in eigenen subjektiven Rechten Verletzten gleichen Typus existiert, die grundsätzlich alle eine Einzelklagebefugnis gem. § 42 Abs. 2 VwGO (analog) hätten, so dass es sinnvoll erscheint, den Sachverhalt in einem gemeinsamen Prozess zu lösen. Die aufgrund desselben Sachverhalts gleich oder ähnlich betroffenen Individuen können sich in einem Prozeß zusammenschließen; die Klagen, bei denen dieselbe Klageart statthaft sein muss, werden gebündelt. Es können ein oder mehrere Mitglieder einer Personengruppe unter bestimmten Voraussetzungen und unter Zustimmung des Gerichts zur Wahrung der Rechte aller Mitglieder der Gruppe auftreten. Streitgegenstand der Klage ist jedoch - und dies ist das Besondere an der Gruppenklage - die Rechtsbeeinträchtigung, die alle Mitglieder der durch die Normverletzung betroffenen Gruppe erlitten haben (inklusive des/der Kläger(s)). Die Rechtskraft wirkt daher nicht nur inter partes, sondern gegenüber sämtlichen Gruppenmitgliedern. Das Urteil bindet alle Mitglieder der Gruppe, die nicht ausdrücklich um Ausschluß aus der Gruppe gebeten haben (»opt-out-System «). ${ }^{69}$

Es handelt sich um Massenverletzungen mit Einzelschäden. Massenverletzungen sind Fälle, in denen ein schädigendes Ereignis, eine Handlung oder Unterlassung zu einer Rechtsverletzung führt, die breit gestreut ist und bei einer Vielzahl von Personen Abwehr-, Beseitigungs- oder Restitutionsansprüche auslöst. Da die betroffenen Rechte auch individuell geltend gemacht werden könnten, erschöpft sich der ökonomische Grund für solche Klagen in der Beeinflussung der Kostenseite. Durch die Bündelung der Mittel und damit die Erhöhung der »Schlagkraft« sowie durch die Einsparung von

68 Es ist zudem davon auszugehen, dass die Einführung von Massenklagen auf weniger Widerstand stoßen würde. Bislang sind direkte Volksrechte auf Bundesebene nur in Art. 29 Abs. 3 GG zu finden. Am 07. Juni 2002 ist im Bundestag das Gesetz für die Einführung von Volksinitiativen, Volksbegehren und Volksentscheide gescheitert. Für den Entwurf stimmten SPD, Grüne, PDS und einige Abgeordnete der FDP. Die für eine Grundgesetzänderung erforderliche Zweidrittel-Mehrheit wurde jedoch aufgrund der Ablehnung durch CDU/CSU und große Teile der FDP nicht erreicht.

$69 \mathrm{Zu}$ den Voraussetzungen der Gruppenklage im Zivilrecht in den USA, siehe ausführlich Koch, Kollektiver Rechtsschutz im Zivilprozeß. 
Gerichts- und Transaktionskosten werden die erwarteten (individuellen) Kosten gesenkt. Dies ist zum einen darauf zurückzuführen, dass die erwarteten Kosten durch alle Kläger geteilt werden. Zum anderen wird die Erfolgschance der Klage durch die gebündelte »Schlagkraft« erhöht, was seinerseits dazu führt, dass aufgrund des AllesOder-Nichts-Prinzips der Kostenregelung die erwarteten Gerichts- und Anwaltskosten gesenkt werden.

Der erste Grund, die Bündelung der Mittel und der Schlagkraft der betroffenen Individuen, sollte nicht gering geschätzt werden. Empirische Erfahrungen bezüglich der Erfolgschancen der Gruppenklage gibt es in Deutschland nicht. Als einzige Möglichkeit der Bündelung von Verwaltungsgerichtsverfahren ist in Deutschland die Streitgenossenschaft gemäß $\S 64$ VwGO vorgesehen, die auf die zivilprozeßrechtliche Streitgenossenschaft ( $\$ 59$ - 63 ZPO) verweist. Ganz im Gegensatz zum europäischen und außereuropäischen Ausland, in dem die Gruppenklage (bzw. »class action«) zugelassen ist, wurde in Deutschland kein Massenverfahren in Streitgenossenschaft von der klagenden Gruppe gewonnen. ${ }^{70}$ Die Verfahren wurden entweder außergerichtlich gelöst oder aber aus prozessualen Gründen abgewiesen. »Anzunehmen, dass es solche Fälle (die der Massenverletzungen; A.v.A.) in Deutschland nicht gäbe, ist ebenso weltfremd wie die Annahme, der Vergleichsweg würde so viel effektiver beschritten als im Ausland. « ${ }^{71}$ Soweit nur Rechtsfragen, nicht aber Sachfragen geklärt werden müssen, ist die Gruppenklage aus Kostengründen nicht so dringend, ${ }^{72}$ da davon ausgegangen werden kann, dass die Entscheidung in einem ersten Prozeß wegen der faktischen Präjudizwirkung von den Behörden beachtet werden wird und somit Kosten nur bei diesem einmaligen Zwei-Parteien-Prozeß anfallen. Hier taucht dann allerdings die Gefahr des Trittbrettfahrens auf, da die Entscheidung des Gerichts in der jeweiligen Rechtsfrage als öffentliches Gut wirkt. Daher werden nur diejenigen, die eine starke Präferenz für eine gerichtliche Entscheidung haben, überhaupt klagen, ${ }^{73}$ die anderen aber sich eben auf die Präjudizklage und die folgende Beachtung der gerichtlichen Entscheidung durch die Verwaltung verlassen. Es kann zu einer Ausbeutung der »Großen « durch die »Kleinen « kommen. ${ }^{74}$ Alle diese Überlegungen werden natürlich ceteris paribus angestellt, d.h. andere Faktoren, wie beispielsweise die Art der Bezahlung von Anwälten mit ihren jeweiligen Anreizwirkungen, werden außer Betracht gelassen.

Auch der zweite Grund, die Einsparung von Kosten, ist bei Gruppenklagen ein nicht zu vernachlässigender Faktor. Es kann davon ausgegangen werden, dass es sowohl auf

70 Hass, Die Gruppenklage: Wege zur prozessualen Bewältigung von Massenschäden, S. 19.

71 Hass, Die Gruppenklage, S. 19.

72 Dies gilt zumindest volkswirtschaftlich, auch wenn für das klagende Individuum kein Unterschied in den erwarteten Kosten besteht.

73 In den USA ist es genau aus diesem Grund im Kartellrecht - eingedenk der Überwachungsdefizite der Bundesbehörden - den Klägern möglich, den dreifachen Schaden geltend zu machen. Dies ist ein klares Beispiel von Stimulation privater Klagen zur Rechtsdurchsetzung. In Deutschland scheint immer noch die Auffassung zu herrschen, dass das Kartellrecht nicht den Verbraucher schützen wolle. Siehe dazu eingehend Kötz, Klagen Privater im öffentlichen Interesse, S. 89.

74 Kirsch, Neue Politische Ökonomie, S. 152. »Groß« und »Klein« bezieht sich auf die Präferenzintensität des Individuums. 
Beklagtenseite, als auch auf Klägerseite, ganz zu schweigen von den Gerichtskosten, zur Einsparung von Transaktions- und Informationskosten kommen wird. Die Bündelung der Prozesse erlaubt es, nur noch einen Anwalt zu beauftragen, anstatt dass jedes Individuum sich um entsprechende anwaltliche Vertretung kümmern muß. Für das einzelne Individuum ist es sowohl einfacher als auch billiger, sich an einen solchen Prozeß anzuhängen. Die Beklagtenseite kann sich ihrerseits auf die Gesamtvertretung einstellen. Die Gerichte haben nur noch einen Prozeß anstatt vieler zu bearbeiten. Dies erlaubt es, den Prozeß gründlicher zu führen und bezüglich der Sachfragen eingehendere Untersuchungen anzustellen, die sich für einen einzelnen Prozeß nicht lohnen würden.

\section{III. Ökonomischer Sinn von Verbandsklagen}

Die Verbandsklage ${ }^{75}$ ist eine Klageart, bei der bestimmte Zweckverbände im eigenen Namen, aber im Interesse von anderen einen Prozeß führen können. Sie unterscheidet sich von der Gruppenklage dadurch, dass in der Gruppenklage subjektive Rechtskraftwirkung gegeben ist, während der Verbandskläger nicht in Konkurrenz zum einzelnen Rechträger tritt. ${ }^{76}$ Hier sind sie jedoch nicht notwendigerweise in eigenen subjektiven Rechten betroffen, vielmehr können durch die Verbandsklage auch diffuse Interessen ihrer Mitglieder oder der Allgemeinheit geltend gemacht werden. Der Verband handelt dann im Namen aller (potentiell) Betroffenen als Agent für diese gegenüber dem Staat. Verbandsklagen erlauben daher die Geltendmachung von Interessen ihrer Mitglieder (egoistische Verbandsklagen) ${ }^{77}$ oder auch Interessen der Allgemeinheit (altruistische oder ideelle Verbandsklagen). ${ }^{78}$ Typischerweise handelt es sich um (potentielle) Schäden, die zwar bei Einzelnen, aber gehäuft auftreten. Im öffentlichen Recht ist die Verbandsklage auf Bundesebene (nur) im Umweltrecht erst vor kurzem eingeführt worden. ${ }^{79}$

$75 \mathrm{Zu}$ einem rechtsvergleichenden Überblick über die Verbandsklage in Europa mit den entsprechenden EU-Richtlinien (Richtlinie 98/27) sowie grenzüberschreitenden Verbandsklagen und dem dort anwendbaren Recht, siehe Koch, Verbandsklage in Deutschland und Europa, S. 413ff. Zu der in Deutschland zur Verbandklage geführten Diskussion, siehe Masing, Die Mobilisierung des Bürgers, S. 121f. m.w.N.

76 Vgl. Koch, Verbandsklage in Deutschland und Europa, S. 413ff., S. 424.

77 Die egoistische Verbandsklage kann lokalen Bürgerinitiativen zusätzlich zu Beteiligungsund Anhörungsrechten an den für sie relevanten Verwaltungsverfahren auch Klagerechte gegen die im Verfahren ergehenden Entscheidungen geben; sie bündelt damit lediglich Interessen von Individuen, die nach bisherigem Recht Beteiligungs- und Klagerechte besitzen. Vgl. Koch, Alternativen zum Zweiparteiensystem, S. 328ff. zu der Frage wessen Recht in wessen Namen bei Verbandsklagen geltend gemacht wird.

78 Die altruistische Verbandsklage macht bestimmte Verbände zu »Hütern der Gesetzmäßigkeit der Verwaltung «. Die Verbände sollen durch Beteiligung am Verwaltungsverfahren und notfalls Erhebung einer Beanstandungsklage die Einhaltung der einschlägigen Gesetze kontrollieren können.

79 Durch das am 15. November 2001 beschlossene BNatSchNeuregG (BT-Drucksache 14/ 7469) wurde u.a. in den $\S \S 58-61$ BNatSchNeuregG eine Verbandsklage von Umweltschutzverbänden auf Bundesebene eingeführt. Auf Länderebene sehen bspw. das bremische Naturschutzgesetz vom 17.9.1979 in den $\S \S 43,44$ sowie $\S 36$ des hessischen Natur- 
Betrachten wir zunächst die egoistische Verbandsklage. Diese kommt in Betracht, wenn eine Vielzahl von Bürgern in ihren subjektiven Rechten geschädigt wird. ${ }^{80}$ In der Regel klagen diese aber nicht, da entweder ihnen selbst ihr Schaden im Einzelfall als zu gering erscheint, es sich um Bagatellschäden handelt oder sie, wie in der Regel, gar nicht genügend über die Schäden und ihre rechtlichen Implikationen informiert sind. Soweit durch eine Verbandsklage die Beeinträchtigung subjektiver Rechte geltend gemacht wird, kann weitgehend auf die ökonomische Begründung der Gruppenklage verwiesen werden. Denn dann gibt es grundsätzlich für den Einzelnen die Möglichkeit, auch individuell zu klagen. Die Wahrnehmung der subjektiven Rechte gegenüber dem Staat ist also nicht de iure schon ausgeschlossen. Die mangelnde Wahrnehmung des Rechts beruht vielmehr auf tatsächlichen Gründen. Manchmal werden in solchen Fällen deshalb Bürgerinitiativen »unter dem Etikettenschwindel von Individualrechtsschutzverfahren $\ll{ }^{81}$ aktiv, indem sie den Individualkläger durch materielle und informationelle Ressourcen unterstützen. Dies ist der Transparenz des Verwaltungsprozeßrechtes aber nicht dienlich, da hier eine dem Wortlaut des Gesetzes widersprechende prozeßrechtliche Praxis etabliert wird.

Ebenso ist die Verbandsklage aber auch denkbar für Fälle, in denen Einzelne nicht in subjektiven Rechten geschädigt sind (altruistische Verbandsklage), entweder weil der Schadensfall noch nicht eingetreten ist oder aber weil es sich um diffuse Interessen handelt, die in der geltenden Systematik nicht individuell einklagbar sind.

Bei den Verbandsklagen trägt der Verband die erwarteten Kosten der Klage. Für die betroffenen Individuen fallen daher keine Kosten an, außer bei den Mitgliedern des Verbandes, auf welche die Kosten umgelegt werden. Aber auch für sie sind die erwarteten Kosten nur ein Bruchteil von dem, was bei einer Individualklage aufgebracht werden müßte. Die Ausführungen zur Gruppenklage gelten entsprechend. Auch kommt bei der Verbandsklage gegenüber der Gruppenklage als Vorteil hinzu, dass ein Verband als spezieller und institutioneller (also nicht als nur ad hoc gebildeter) Interessenzusammenschluß ein besonderes Fachwissen in den betreffenden Sach- und Rechts-

schutzgesetzes vom 19.9.1980 ein solches Klagerecht vor. Ebenso haben die neuen Bundesländer die Verbandsklage im Umweltrecht eingeführt. Im schweizerischen Recht existiert die Verbandsklage, allerdings ist sie auf Feststellungs- und Unterlassungsbegehren beschränkt und ermöglicht keine Schadensersatzforderungen. Im französischen Recht existiert die »action collective« oder »action syndicale« für Naturschutzverbände oder auch zur Bekämpfung von Rassendiskriminierung. Die juristische Struktur und der faktische Gebrauch sind von Land zu Land unterschiedlich. Siehe dazu ausführlich Koch, Prozeßführung im öffentlichen Interesse und Koch, Verbandsklage in Deutschland und Europa, speziell auf die unterschiedliche Zulassung des Rechtsschutzbegehrens (Schadensersatz, Unterlassung, Beseitigung, Vertragserfüllung, Anfechtung), S. 417.

80 Im Zivilrecht ist die Notwendigkeit von Verbandsklagen etwa dann anerkannt, wenn eine Vielzahl von Verbrauchern in ihren Rechten betroffen sein können. So ist die Verbandsklage auf Bundesebene zugelassen für Verbraucherschutzverbände in § 3 UKLG und in $\S 13$ UWG. Siehe zum deutschen Recht und rechtsvergleichend auch Koch, Verbandsklage in Deutschland und Europa, S. 413ff.

81 So Kötz, Klagen Privater im öffentlichen Interesse, S. 91ff. 
fragen vorweisen kann. ${ }^{82}$ Damit wird die Informationsasymmetrie, die in der Regel bei Einzelklägern gegenüber dem Staat besteht (auch wenn sie als Gruppe klagen), zumindest teilweise aufgehoben. Verbände haben in der Regel die entsprechende Expertise und die Fachleute, die in komplizierten Fragen kompetent den Klägerstandpunkt vertreten können. Verbände haben auch in der Öffentlichkeit eine stärkere Position und damit die Möglichkeit, Gelder zu beschaffen, die ansonsten nicht verfügbar wären.

Worin besteht aber für den Verband der Anreiz zu klagen? Seit Olsons »Logik des kollektiven Handelns $\ll^{83}$ ist bekannt, dass sich große Gruppen, die ein öffentliches Gut anbieten wollen, nur schwer zusammenschließen. Insbesondere wenn der Einzelne für das betreffende Gut keine starke Präferenz hat bzw. die Nutzenbeeinträchtigung und Externalität nicht stark ins Gewicht fällt, wird es zu keinem Zusammenschluß der Betroffenen kommen, und ihre Interessen werden nicht wahrgenommen. Dies ist gerade der Fall bei Umweltschutzbelangen, aber ebenso bei Steuerzahlern oder Konsumenten. ${ }^{84}$ Bislang sieht man, dass in den Ländern, in denen eine Verbandsklage de lege lata besteht, die Organisationen diese Prozeßform wählen, wenn sie ihre (potentiellen) Mitglieder mobilisieren, die Bindung zu ihren Mitgliedern verstärken oder neue Mitglieder werben wollen. Dies ist vor allem bei neueren und kleinen Organisationen der Fall. ${ }^{85}$ Auch wenn hier wieder Bedenken wegen des Trittbrettfahrerproblems ${ }^{86}$ geltend gemacht werden können, so haben die Verbände dennoch durch die Öffentlichkeitswirkung und Reputation einer solchen Verbandsklage ein Interesse daran, Klagen durchzuführen. Das Führen eines Rechtsstreits zur Durchsetzung gebündelter Interessen kann - teilweise unabhängig von dem Ausgang des Rechtsstreits - der Organisation Legitimation verschaffen, Erwartungen an die Politiker wecken und das gemeinsame Interesse der Betroffenen deutlich machen.

Welche Kosten sind gesamtgesellschaftlich bei der Einführung einer Verbandsklage zu erwarten? Zum einen würden Kosten und Verwaltungsaufwand bei der Anerkennung von Verbänden, die ein Klagerecht beantragen, entstehen. ${ }^{87}$ Diese Kosten sind einmalig und vermutlich nicht sehr hoch. Demgegenüber sind Entlastungen zu erwarten, da sich der Aufwand der Vollzugsbehörden verringert. Die Verbandsklage gibt den Bürgern, die sich organisieren, die Möglichkeit, bei Betroffenheit durch externe Effekte auch Beteiligte zu werden und so die Internalisierung zu fördern. Damit kön-

82 Die Verbände müssen in den Gesetzen, die bislang eine Verbandsklage vorsehen, staatlicherseits anerkannt sein, um ein Klagerecht zu bekommen.

83 Olson, Die Logik des kollektiven Handelns.

84 Olson selbst belegt dies am Beispiel von Arbeitnehmerinteressen. Die Gewerkschaften müssen in der Regel zusätzlich private Güter anbieten, um Anreize für die Mitgliedschaft zu setzen und überhaupt bestehen zu können.

85 Siehe Groenendijk, Die Wahrnehmung gebündelter Interessen im Zivilprozeß, S. 261 m.w.N.

86 Nichtmitglieder können eventuell von der Entscheidung in einer Rechtsfrage profitieren.

87 Je strenger die Anerkennungsvoraussetzungen für Verbände sind, desto größer wird allerdings die Gefahr, dass beispielsweise Bürgerinitiativen keine Klagebefugnis erhalten. Dann wäre die Beteiligung von Betroffenen allerdings nicht immer gewährleistet. Siehe zu der Frage der Anerkennung von Verbänden auch Koch, Verbandsklage in Deutschland und Europa, S. 430f. 
nen soziale Probleme in der Form von Externalitäten von denen geltend gemacht werden, die davon betroffen sind, ohne dass darauf gewartet werden muß, dass die Verwaltung tätig wird, bzw. rechtmäßig tätig wird. Zudem ist zu erwarten, dass allein die Klagemöglichkeit einen präventiven Effekt aufweist, weil durch sie eine Art »Drohpotential« geschaffen wird, was die Verwaltung dazu anhält, die Rechtmäßigkeit von Entscheidungen oder Unterlassungen selbst genauer zu prüfen. ${ }^{88}$

Verbandsklagen sind insbesondere für alle ad hoc schwer organisierbaren Interessen denkbar, also Umweltschutz-, Konsumenten-, Steuerzahler-, Mieter-, Autofahrerinteressen etc.

\section{IV. Ökonomischer Sinn von Popularklagen}

Die Popularklage ist eine Klageart, bei der ein Einzelner, der nicht notwendigerweise in eigenen subjektiven Rechten betroffen ist, gegen (oder für) eine staatliche Maßnahme in Form eines materiellen Gesetzes klagen kann, wenn er das Gesetz (oder die Unterlassung des Erlasses) für rechtswidrig bzw. verfassungswidrig hält. ${ }^{89}$ Die Verfassung des Freistaates Bayern sieht eine solche Popularklage gegen Gesetze und Verordnungen in Art. 98 S. 4 BayVerf, § 55 II Nr. 1 BayVerfGG vor; auf Bundesebene

88 In der juristischen Literatur werden Verbandsklagen seit längerem diskutiert. Die Gegenargumente sehen in der Regel folgendermaßen aus (stellvertretend für viele, siehe Weyreuther, Verwaltungskontrolle durch Verbände, Argumente gegen die verwaltungsgerichtliche Verbandsklage im Umweltrecht, 1975: Es bestehe kein Bedürfnis für Verbandsklagen; die Eignung der Verbände als Legitimationsträger sei nicht dargetan; die Verbandsklage beeinträchtige die Rechtsposition von Drittbetroffenen (also denen, denen ein Recht nicht zuerkannt wird, beispielsweise ein Atomkraftbetreiber) in unerträglicher Weise; die Verbandsklage führe tendenziell zu einem sachwidrigen und verfassungsrechtlich bedenklichen Bruch mit dem gegenwärtigen, am individuellen Rechtsschutz ausgerichteten System der Verwaltungsgerichtsbarkeit. Vgl. auch die Besprechung dieser Argumente in Koch, Prozeßführung im öffentlichen Interesse, S. $307 \mathrm{ff}$.

89 Auch die der Popularklage ähnlichen Normenkontrollverfahren wie bis 1996 § 47 VwGO, die vom Bürger beantragt werden können, ohne in einem subjektiven Recht verletzt zu sein, sind auf Bundesebenen nicht mehr vorgesehen.

In den USA existiert die Popularklage als »citizen suit« oder auch »tax-payers suit«, vgl. ausführlich dazu Koch, Prozeßführung im öffentlichen Interesse, insbes. S. 53ff. Sie ist besonders geläufig im Wahlrecht und Naturschutzrecht, im Patent- und Warenzeichenrecht sowie im Kartellrecht. Dies sind auch klassisch die Gebiete, in denen genau die hier geführte Argumentation zutrifft, nämlich Gebiete mit massenhaft, aber geringfügig auftretenden Schäden, die keine subjektiven Rechte betreffen, in denen aber zur Überwachung der Einhaltung von Rechtsvorschriften, einem »privaten Staatsanwalt« ein Anreiz zur Kontrolle gegeben werden soll.

$\mathrm{Zu}$ einer Kritik der neuen, die Klagebarrieren abbauenden Entscheidung des US-Supreme Court im Umweltrecht Friends of Earth, Inc. v. Laidlaw Environmental Services, Inc., 528 U.S. 167 (2000), die eine Popularklagebefugnis schon bei der Verletzung von technischen Vorschriften, ohne das ein Schaden nachgewiesen werden muss, zulässt, siehe Adler, Stand or Deliver: Citizen Suits, Standing, and Environmental Protection, S. 39ff. Zu der vorangehenden (nun aufgehobenen) Entscheidung Lujan v. National Wildlife Fed’n, 497 U.S. 871 (1990), siehe Sunstein, What's Standing after Lujan? Of Citizen Suits, "Injuries", and Article III, Michigan Law Review 91, 1992, S. $163 \mathrm{ff}$. 
existiert sie in allgemeiner Form im öffentlichen Recht nicht. ${ }^{90}$ Hier wird deutlich, dass sich die Popularklage auch auf die Legislativebene beziehen kann, wenn nämlich das angegriffene materielle Gesetz von der Legislative erlassen wurde. Die Popularklage wirkt dann genau wie eine Ablehnungsinitiative. Hier konzentriert sich das Interesse jedoch auf die Kontrolle der Verwaltung, weshalb im folgenden nur Klagen gegen die legislative Tätigkeit der Verwaltung behandelt werden. Die Popularklage gibt einem Einzelnen das Recht, quasi stellvertretend für andere, subjektive Rechte oder auch diffuse Interessen geltend zu machen. Die Popularklage ist zwar von ihrer juristischen Form her ein Zwei-Parteien-Prozeß, aber da sie sich gegen materielle Gesetze, also generell-abstrakte Regelungen, wendet und daher nicht nur inter partes wirkt, hat sie de facto den Charakter einer Massenklage in Form einer Normenkontrollklage. Sie ist damit die ausgeprägteste Form der Klagen Privater im öffentlichen Interesse, mithin also die private Bereitstellung eines rein öffentlichen Gutes.

Die Kosten für das Individuum, eine Individualklage anzustrengen, sind, wie oben bereits ausgeführt, hoch. Der erwartete Nutzen ist niedrig, wenn das Individuum nicht in einem subjektiven Recht betroffen ist. Ökonomisch betrachtet, sind daher die Anreize für ein Individuum, eine Popularklage anzustrengen, außerordentlich gering. In welchen Fällen bestehen für das Individuum dennoch Anreize zu klagen, wenn es nicht in einem subjektiven Recht betroffen ist? Ein Anreiz besteht wohl nur dann, wenn eine starke Präferenzintensität für die Rechtmäßigkeit von materiellen Gesetzen besteht. Eine solche Präferenz, als »Institutionenschützer ${ }^{91}{ }^{2}$ zu fungieren, wird in aller Regel nur bei Individuen, die sich profilieren wollen, beispielsweise politischen Unternehmern oder Medienvertretern, angenommen werden können. Politische Unternehmer suchen, wie alle Unternehmer, Marktlücken; also im politischen Bereich unter anderem unbeachtete Externalitäten. Dabei kann es sich um subjektive Rechte anderer oder auch diffuse Interessen (objektives Recht) handeln. Bei politischen Unternehmern kann eine starke Präferenz angenommen werden, Defizite im Vollzug von Gesetzen oder die Rechtswidrigkeit von Gesetzen, aufzudecken.

Popularklagen, mit ihrem Charakter als Normenkontrollantrag, sind ein wirksames und präventives Instrument zur Kontrolle der Verwaltung. Sie geben einzelnen Bürgern das Recht, gegen als rechtswidrig empfundene materielle Gesetze die Gerichte anzurufen. Dies ist eine wirksame Kontrolle der Legislativtätigkeit der Verwaltung, da oftmals viel Zeit vergeht, bis sich ein Kläger findet, der durch das jeweilige Gesetz in einem subjektiven Recht betroffen ist und auch klagt. Popularklagen sind ein direktes Volksrecht auf Verwaltungsebene in dem Sinne von Ablehnungsinitiativen, weil die Rechtmäßigkeit der legislativ tätigen Verwaltung überprüft werden kann, ohne dass die Einzelnen selbst direkt in ihren subjektiven Rechten betroffen sind. Aufmerksame Bürger können, auch ohne auf eigene subjektive Rechtsbetroffenheit warten zu müssen, die Rechtmäßigkeit des Gesetzes überprüfen lassen. Es ist nicht ersichtlich, warum ein Individuum, dessen Präferenz für ein diffuses Interesse so stark ist, dass es

90 Die Popularklage ist bei Nichtigkeits- und Löschungsklagen in den Fällen der $\S \S 22,81$ PatG und des § 55 II Nr. 1 MarkenG zugelassen.

91 So Koch, Die Funktionäre des Sozialschutzes im Prozeß, S. 400. 
die Nachteile und Kosten eines Prozesses auf sich zu nehmen bereit ist, keine Klagemöglichkeit bekommen sollte.

Zusammenfassend soll nochmals gezeigt werden, welche Rechtsschutzlücken bei der externen Kontrolle der Verwaltung bestehen und wie dies de lege ferenda geändert werden könnte.

\begin{tabular}{|l|ll|}
\hline & subjektive Rechte & objektives Recht \\
\hline de lege lata & $\begin{array}{l}\text { Betroffenheit in eigenem } \\
\text { subjektiven Recht } \\
\text { Individualklage }\end{array}$ & \\
\hline de lege ferenda & $\begin{array}{l}\text { Betroffenheit in eigenem } \\
\text { subjektiven Recht }\end{array}$ & $\begin{array}{l}\text { Betroffenheit von } \\
\text { diffusen Interessen }\end{array}$ \\
& $\begin{array}{l}\text { Gruppenklage/ } \\
\text { Verbandsklage }\end{array}$ & $\begin{array}{l}\text { Verbandsklage/ } \\
\text { Popularklage }\end{array}$ \\
& $\begin{array}{l}\text { Betroffenheit von subjektiven } \\
\text { Rechten allgemein }\end{array}$ & \\
& $\begin{array}{l}\text { Popularklage/ } \\
\text { Verbandsklage }\end{array}$ & \\
\hline
\end{tabular}

Rechtsschutzlïcken (Abb. 5)

\section{Schlussbemerkung}

Nach institutionenökonomischer Betrachtungsweise besteht zwischen Staat und Bürgern ein Prinzipal-Agenten-Problem. Auf Rechtssetzungsebene kann die Kontrolle des Agenten durch Wahlen durch direkte Volksrechte ergänzt werden. Dies ist jedoch nicht die einzige Möglichkeit demokratischer Kontrolle oder auch zivilgesellschaftlicher Elemente, auch wenn in aller Regel demokratische Partizipation nur auf der Rechtssetzungsebene analysiert wird. Vernachlässigt wird Partizipation und Kontrolle zumeist auf der Rechtsdurchsetzungsebene, also auf der Ebene der Verwaltung. Das Problem wird aber dann um so wichtiger, je mehr Entscheidungskompetenz die Legislative an die Exekutive delegiert, insbesondere, wenn die Verwaltung vermehrt Rechtssetzungskompetenz erhält.

Die unzureichende externe Kontrolle der Verwaltung - rechtlich wegen mangelnder Klagemöglichkeiten oder tatsächlich wegen rationaler Abstinenz der Bürger bei externen Effekten - ist ein ubiquitäres Phänomen der Massengesellschaft. Eine Möglichkeit der direkten Verwaltungskontrolle durch Bürger sind nicht nur Informationsrechte, sondern auch Klagen, insbesondere Massenklagen. De lege lata hält das deutsche Verwaltungsprozessrecht aber an der Idee des »bourgeois« fest, gemäß welcher der Bürger nur seinen privaten, individuellen Anspruch, aber eben nicht als Sachwalter der Allgemeinheit die Gesetzmäßigkeit der Verwaltung geltend macht. Die gegebenen 
schwachen Anreizstrukturen bei Individualklagen sind ein starkes Argument für die Einführung von Massenklagen, damit eine effektive Verwaltungskontrolle stattfinden kann. Weiterhin ist über die Aufschnürung politischer Pakete durch Massenklagen nachzudenken, denn funktional sind Massenklagen und direkte Volksrechte verwandt. Massenklagen teilen viele Vorzüge der direkten Volksrechte, jedoch nicht alle ihre Nachteile.

Daher sollte über eine wirksamere Verwaltungskontrolle durch eine Erweiterung der Klagebefugnis einerseits und die Stimulierung privater Klageinitiative andererseits verstärkt nachgedacht werden. Aus den genannten Gründen wird in diesem Aufsatz für die Einführung von Massenklagen plädiert, die nicht nur die Beachtung subjektiver Rechte bei Massenschäden, sondern auch die Beachtung des objektiven Rechts durch die Verwaltung fördern. Auf diese Weise kann eine lückenlosere Kontrolle der Agenten durch die Bürger erwartet werden.

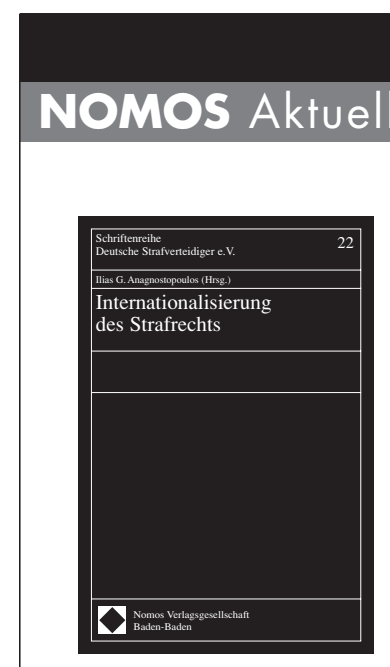

Ilias G. Anagnostopoulos (Hrsg.) Internationalisierung des Strafrechts 2003, 151 S., brosch., 29, $-€$, ISBN 3-7890-8378-X

(Schriftenreihe Deutsche Strafverteidiger e.V., Bd. 22)

Die Aufsätze dieses Bandes sind aktuellen Fragen der Internationalisierung des Strafrechts gewidmet.

Der Schutz der Menschenrechte im Strafverfahren, die internationale Strafgerichtsbarkeit, die internationale Rechtshilfe in Strafsachen, Geldwäsche, der Schmuggel innerhalb der Europäischen Union, der Schutz der finanziellen Interessen der Europäischen Gemeinschaften, ne bis in idem, Strafrecht und Internet, werden von den Autoren, die etablierte Strafrechtstheoretiker und -praktiker sind, schwerpunktmäBig dargestellt.

Prof. Dr. jur. llias G. Anagnostopoulos lehrt Straf- und Strafprozessrecht an der Universität Athen und ist als Strafverteidiger in Griechenland und international tätig. 